Article

\title{
R-Matrices, Yetter-Drinfel'd Modules and Yang-Baxter Equation
}

\section{Victoria Lebed}

Institut de Mathématiques de Jussieu-Paris Rive Gauche, UMR7586, Bâtiment Sophie Germain, Case 7012, 75205 PARIS Cedex 13, France; E-Mail: lebed@math.jussieu.fr; Tel.: +33-6-28-59-28-61

Received: 14 August 2013; in revised form: 28 August 2013 / Accepted: 30 August 2013 /

Published: 5 September 2013

\begin{abstract}
In the first part we recall two famous sources of solutions to the Yang-Baxter equation-R-matrices and Yetter-Drinfel'd (=YD) modules-and an interpretation of the former as a particular case of the latter. We show that this result holds true in the more general case of weak R-matrices, introduced here. In the second part we continue exploring the "braided" aspects of YD module structure, exhibiting a braided system encoding all the axioms from the definition of YD modules. The functoriality and several generalizations of this construction are studied using the original machinery of YD systems. As consequences, we get a conceptual interpretation of the tensor product structures for YD modules, and a generalization of the deformation cohomology of YD modules. This homology theory is thus included into the unifying framework of braided homologies, which contains among others Hochschild, Chevalley-Eilenberg, Gerstenhaber-Schack and quandle homologies.
\end{abstract}

Keywords: Yang-Baxter equation; braided system; Yetter-Drinfel'd module; R-matrix; braided homology

\section{Introduction}

The Yang-Baxter equation (YBE) is omnipresent in modern mathematics. Its realm stretches from statistical mechanics to quantum field theory, covering quantum group theory, low-dimensional topology and many other fascinating areas of mathematics and physics. The attemps to understand and classify all solutions to the Yang-Baxter equation (often referred to as braidings, since they provide representations of braid groups) have been fruitless so far. Nevertheless we dispose of several methods of producing vast families of such solutions, often endowed with an extremely rich structure.

In Section 2 we review two major algebraic sources of braidings, which have been thouroughly studied from various viewpoints over the past few decades. The first one is given by $R$-matrices for 
quasi-triangular bialgebras, dating back to V.G. Drinfel'd's celebrated 1986 ICM talk [1]. The second one comes from Yetter-Drinfel'd modules (=YD modules) over a bialgebra, introduced by D. Yetter in 1990 under the name of "crossed bimodules" (see [2]) and rediscovered later by different authors under different names (see for instance the paper [3] of S.L. Woronowicz, where he implicitely considers a Hopf algebra as a YD module over itself). All these notions and constructions are recalled in detail in Sections 2.2 and 2.3.

Note that we are interested here in not necessarily invertible braidings; that is why most constructions are effectuated over bialgebras rather than Hopf algebras, though the latter are more current in literature. Section 3.2 contains an example where the non-invertibility does matter.

It was shown in S. Montgomery's celebrated book ([4], 10.6.14) that R-matrix solutions to the YBE can be interpreted as particular cases of Yetter-Drinfel'd type solutions. We recall this result and its categorical version (due to M. Takeuchi, cf. [5]) in Section 2.4. Our original contribution consists in a generalization of this result: we introduce the notion of weak $R$-matrix for a bialgebra $H$ and show it to be sufficient for endowing any $H$-module with a YD module structure over $H$ (cf. the charts on p. 456).

In Section 3 we explore deeper connections between YD modules (and thus R-matrices, as explained above) and the YBE. We show that YD modules give rise not only to braidings, but also to higher-level braided structures, called braided systems. Here we briefly explain this concept after a short recapitulation of two category-theoretic viewpoints on the YBE-a "local" and a "global" ones.

The first one is rather straightforward: in a strict monoidal category $\mathcal{C}$, one looks for objects $V$ and endomorphisms $\sigma$ of $V \otimes V$ satisfying (YBE). Such $V$ 's are called braided objects in $\mathcal{C}$. A more categorical approach consists in working in a "globally" braided monoidal category, as defined in 1993 by A. Joyal and R.H. Street ([6]). Concretely, a braiding on a monoidal category is a natural family of morphisms $\sigma_{V, W}: V \otimes W \rightarrow W \otimes V$ compatible with the monoidal structure, in the sense of Equations (2) and (3). Every object $V$ of such a category is braided, via $\sigma_{V, V}$. See [5] for a comparison of "local" and "global" approaches, in particular in the the context of the definition of braided Hopf algebras. Well-known braidings on the category of modules over a quasi-triangular bialgebra and in that of YD modules over a bialgebra are recalled in Sections 2.2 and 2.3.

Now, the notion of braided system in $\mathcal{C}$ is a multi-term version of that of braided object: it is a family $V_{1}, \ldots, V_{r}$ of objects in $\mathcal{C}$ endowed with morphisms $\sigma_{i, j}: V_{i} \otimes V_{j} \rightarrow V_{j} \otimes V_{i}$ for all $i \leqslant j$, satisfying a system of mixed YBEs. This notion was defined and studied in [7] (see also [8]). The $r=2$ case appeared, under the name of Yang-Baxter system (or WXZ system), in the work of L. Hlavatý and L. Šnobl ([9]). See Section 3.1 for details.

In Section 3.2, we present a braided system structure on the family $\left(H, M, H^{*}\right)$ for a YD module $M$ over a finite-dimensional $\mathbb{k}$-bialgebra $H$ (here $\mathbb{k}$ is a field, and $H^{*}$ is the linear dual of $H$ ). Note that several $\sigma_{i, j}$ 's from this system are highly non-invertible. Its subsystem $\left(H, H^{*}\right)$ is the braided system encoding the bialgebra structure, constructed and explored in [8], and related to, but different from, the braided system considered by F.F. Nichita in [10] (see also [11]).

In order to treat the above construction in a conceptual way and extend it to a braided system structure on $\left(H, V_{1}, \ldots, V_{s}, H^{*}\right)$ for YD modules $V_{1}, \ldots, V_{s}$ over $H$, we introduce the concept of Yetter-Drinfel'd system and show it to be automaticaly endowed with a braiding. See Section 3.3 for details, and Section 3.4 for examples. 
In Section 3.5 we show the functoriality and the precision of the above braided system construction. The functoriality is proved by exhibiting the category inclusion (41). Precision means that the described braided system structure on $\left(H, M, H^{*}\right)$ captures all the algebraic information about the YD module $M$, in the sense that each mixed YBE for this system is equivalent to an axiom from the definition of a YD module, and each YD axiom gets a "braided" interpretation in this way.

Section 3.6 contains an unexpected application of the braided system machinery. Namely, it allows us to recover the two tensor product structures for YD modules, proposed by L.A. Lambe and D.E. Radford in [12], from a conceptual viewpoint.

Applying the general braided (co)homology theory from [8,13] (recalled in Section 3.7) to the braided systems above, we obtain in Section 3.8 a rich (co)homology theory for (families of) YD modules. It contains in particular the deformation cohomology of YD modules, introduced by $\mathrm{F}$. Panaite and D. Ştefan in [14]. The results of Section 2.4 give then for free a braided system structure for any module over a finite-dimensional quasi-triangular $\mathbb{k}$-bialgebra $H$, with a corresponding (co)homology theory. Besides a generalization of the Panaite-Ştefan construction, our "braided" tools allow to considerably simplify otherwise technical verifications from their theory. Moreover, our approach allows to consider YD module (co)homologies in the same unifying framework as the (co)homologies of other algebraic structures admitting a braided interpretation, e.g., associative and Leibniz (or Lie) algebras, self-distributive structures, bialgebras and Hopf (bi)modules (see $[8,13]$ ).

The paper is intended to be as elementary and self-contained as possible. Even widely known notions are recalled for the reader's convenience. The already classical graphical calculus is extensively used in this paper, with

$\rightarrow$ dots standing for vector spaces (or objects in a monoidal category),

$\rightarrow$ horizontal gluing corresponding to the tensor product,

$\rightarrow$ graph diagrams representing morphisms from the vector space (or object) which corresponds to the lower dots to that corresponding to the upper dots,

$\rightarrow$ vertical gluing standing for morphism composition, and vertical strands for identities.

Note that all diagrams in this paper are to be read from bottom to top.

Throughout this paper we work in a strict monoidal category $\mathcal{C}$ (Definition 2.1); as an example, one can have in mind the category Vect $_{\mathbb{k}}$ of $\mathbb{k}$-vector spaces and $\mathbb{k}$-linear maps, endowed with the usual tensor product over $\mathbb{k}$.

For an object $V$ in $\mathcal{C}$ and a morphism $\varphi: V^{\otimes l} \rightarrow V^{\otimes r}$, the following notation is repeatedly used:

$$
\varphi^{i}:=\operatorname{Id}_{V}^{\otimes(i-1)} \otimes \varphi \otimes \operatorname{Id}_{V}^{\otimes(k-i+1)}: V^{\otimes(k+l)} \rightarrow V^{\otimes(k+r)} .
$$

\section{Two Sources of Braidings Revisited}

\subsection{Basic Definitions}

Definition 2.1. A strict monoidal (or tensor) category is a category $\mathcal{C}$ endowed with

$\rightarrow$ a tensor product bifunctor $\otimes: \mathcal{C} \times \mathcal{C} \rightarrow \mathcal{C}$ satisfying the associativity condition;

$\rightarrow$ a unit object $I$ which is a left and right identity for $\otimes$. 
We work only with strict monoidal categories here for the sake of simplicity; according to a theorem of S. MacLane ([15]), any monoidal category is monoidally equivalent to a strict one. This justifies in particular parentheses-free notations like $V \otimes W \otimes U$ or $V^{\otimes n}$. The word "strict" is omitted but always implied in what follows.

The "local" categorical notion of braiding will be extensively used in this paper:

Definition 2.2. An object $V$ in a monoidal category $\mathcal{C}$ is called braided if it is endowed with a "local" braiding, i.e., a morphism

$$
\sigma=\sigma_{V}: V \otimes V \rightarrow V \otimes V
$$

satisfying the (categorical) Yang-Baxter equation $(=Y B E)$

$$
\left(\sigma_{V} \otimes \operatorname{Id}_{V}\right) \circ\left(\operatorname{Id}_{V} \otimes \sigma_{V}\right) \circ\left(\sigma_{V} \otimes \operatorname{Id}_{V}\right)=\left(\operatorname{Id}_{V} \otimes \sigma_{V}\right) \circ\left(\sigma_{V} \otimes \operatorname{Id}_{V}\right) \circ\left(\operatorname{Id}_{V} \otimes \sigma_{V}\right)
$$

Following D. Yetter ([2]), one should use the term pre-braiding here in order to stress that non-invertible $\sigma$ 's are allowed; we keep the term braiding for simplicity.

Graphically, the braiding $\sigma_{V}$ is presented as $\zeta$. The diagrammatical counterpart of (YBE), depicted on Figure 1, is then the third Reidemeister move, which is at the heart of knot theory.

Figure 1. Yang-Baxter equation $\longleftrightarrow$ Reidemeister move III.
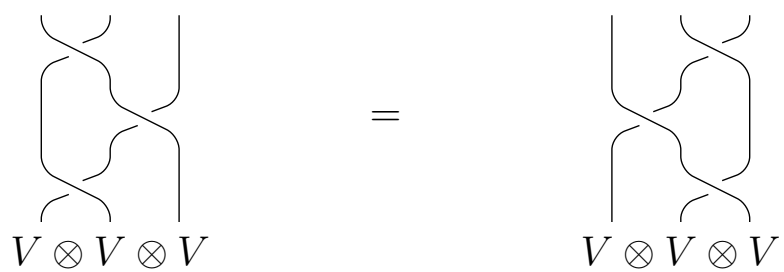

The "global" categorical notion of braiding will also be used here, both for describing the underlying category $\mathcal{C}$ and for constructing and systematizing new braidings:

Definition 2.3. $\rightarrow$ A monoidal category $\mathcal{C}$ is called braided if it is endowed with a braiding (or $a$ commutativity constraint), i.e., a natural family of morphisms $c=\left(c_{V, W}: V \otimes W \rightarrow W \otimes V\right)$ indexed by objects $V, W$ of $\mathcal{C}$, satisfying

$$
\begin{aligned}
& c_{V, W \otimes U}=\left(\operatorname{Id}_{W} \otimes c_{V, U}\right) \circ\left(c_{V, W} \otimes \operatorname{Id}_{U}\right) \\
& c_{V \otimes W, U}=\left(c_{V, U} \otimes \operatorname{Id}_{W}\right) \circ\left(\operatorname{Id}_{V} \otimes c_{W, U}\right)
\end{aligned}
$$

for any triple of objects $V, W, U$. "Natural" means here

$$
c_{V^{\prime}, W^{\prime}} \circ(f \otimes g)=(g \otimes f) \circ c_{V, W}
$$

for all objects $V, W, V^{\prime}, W^{\prime}$ and all morphisms $f \in \operatorname{Hom}_{\mathcal{C}}\left(V, V^{\prime}\right), g \in \operatorname{Hom}_{\mathcal{C}}\left(W, W^{\prime}\right)$.

$\rightarrow$ A braided category $\mathcal{C}$ is called symmetric if its braiding is symmetric:

$$
c_{V, W} \circ c_{W, V}=\operatorname{Id}_{W \otimes V} \quad \forall V, W \in \mathrm{Ob}(\mathcal{C})
$$


The part "monoidal" of the usual terms "braided monoidal" and "symmetric monoidal" are omitted in what follows.

Lemma 2.4. Every object $V$ in a braided category $\mathcal{C}$ is braided, with $\sigma_{V}=c_{V, V}$.

Proof. Take $V^{\prime}=V, W^{\prime}=W=V \otimes V, f=\mathrm{Id}_{V}$ and $g=c_{V, V}$ in the condition (4) expressing naturality; this gives the YBE.

We further define the structures of algebra, coalgebra, bialgebra and Hopf algebra in a monoidal category $\mathcal{C}$ :

Definition 2.5. $\rightarrow$ A unital associative algebra $(=U A A)$ in $\mathcal{C}$ is an object $A$ together with morphisms $\mu: A \otimes A \rightarrow A$ and $\nu: \mathbf{I} \rightarrow A$ satisfying the associativity and the unit conditions:

$$
\begin{aligned}
& \mu \circ\left(\operatorname{Id}_{A} \otimes \mu\right)=\mu \circ\left(\mu \otimes \operatorname{Id}_{A}\right): A^{\otimes 3} \rightarrow A \\
& \mu \circ\left(\operatorname{Id}_{A} \otimes \nu\right)=\mu \circ\left(\nu \otimes \operatorname{Id}_{A}\right)=\operatorname{Id}_{A}
\end{aligned}
$$

A UAA morphism $\varphi$ between UAAs $\left(A, \mu_{A}, \nu_{A}\right)$ and $\left(B, \mu_{B}, \nu_{B}\right)$ is a $\varphi \in \operatorname{Hom}_{\mathcal{C}}(A, B)$ respecting the UAA structures:

$$
\begin{aligned}
& \varphi \circ \mu_{A}=\mu_{B} \circ(\varphi \otimes \varphi): A \otimes A \rightarrow B \\
& \varphi \circ \nu_{A}=\nu_{B}
\end{aligned}
$$

$\rightarrow$ A UAA $(A, \mu, \nu)$ in $\mathcal{C}$ is called braided if it is endowed with a braiding $\sigma$ compatible with the UAA structure. Using notation (1), this can be written as

$$
\begin{aligned}
& \sigma \circ \mu^{1}=\mu^{2} \circ\left(\sigma^{1} \circ \sigma^{2}\right): A^{\otimes 3} \rightarrow A^{\otimes 2} \\
& \sigma \circ \mu^{2}=\mu^{1} \circ\left(\sigma^{2} \circ \sigma^{1}\right): A^{\otimes 3} \rightarrow A^{\otimes 2} \\
& \sigma \circ \nu^{1}=\nu^{2}: A=\mathbf{I} \otimes A=A \otimes \mathbf{I} \rightarrow A^{\otimes 2} \\
& \sigma \circ \nu^{2}=\nu^{1}: A=\mathbf{I} \otimes A=A \otimes \mathbf{I} \rightarrow A^{\otimes 2}
\end{aligned}
$$

$\rightarrow$ A counital coassociative coalgebra $(=\operatorname{coUAA})$ in $\mathcal{C}$ is an object $C$ together with morphisms $\Delta:$ $C \rightarrow C \otimes C$ and $\varepsilon: C \rightarrow$ I satisfying the coassociativity and the counit conditions:

$$
\begin{aligned}
\left(\Delta \otimes \operatorname{Id}_{C}\right) \circ \Delta & =\left(\operatorname{Id}_{C} \otimes \Delta\right) \circ \Delta: C \rightarrow C^{\otimes 3} \\
\left(\varepsilon \otimes \operatorname{Id}_{C}\right) \circ \Delta & =\left(\operatorname{Id}_{C} \otimes \varepsilon\right) \circ \Delta=\operatorname{Id}_{C}
\end{aligned}
$$

A coUAA morphism $\varphi$ between coUAAs is a morphism in $\mathcal{C}$ respecting the coUAA structures.

$\rightarrow$ A coUAA $(C, \Delta, \varepsilon)$ in $\mathcal{C}$ is called braided if it is endowed with a braiding $\sigma$ compatible with the coUAA structure, in the sense analogous to Equations (8)-(11).

$\rightarrow$ A left module over a UAA $(A, \mu, \nu)$ in $\mathcal{C}$ is an object $M$ together with a morphism $\lambda: A \otimes M \rightarrow M$ respecting $\mu$ and $\nu$ :

$$
\begin{aligned}
& \lambda \circ\left(\mu \otimes \operatorname{Id}_{M}\right)=\lambda \circ\left(\operatorname{Id}_{A} \otimes \lambda\right): A \otimes A \otimes M \rightarrow M \\
& \lambda \circ\left(\nu \otimes \operatorname{Id}_{M}\right)=\operatorname{Id}_{M}
\end{aligned}
$$


An $A$-module morphism $\varphi$ between $A$-modules $\left(M, \lambda_{M}\right)$ and $\left(N, \lambda_{N}\right)$ is a $\varphi \in \operatorname{Hom}_{\mathcal{C}}(M, N)$ respecting the $A$-module structures:

$$
\varphi \circ \lambda_{M}=\lambda_{N} \circ\left(\operatorname{Id}_{A} \otimes \varphi\right): A \otimes M \rightarrow N
$$

The category of $A$-modules in $\mathcal{C}$ and their morphisms is denoted by ${ }_{4}$ Mod.

Right modules, left/right comodules over coUAAs and their morphisms are defined similarly.

$\rightarrow$ A bialgebra in a braided category $(\mathcal{C}, \otimes, \mathbf{I}, c)$ is a UAA structure $(\mu, \nu)$ and a coUAA structure $(\Delta, \varepsilon)$ on an object $H$, compatible in the following sense:

$$
\begin{aligned}
\Delta \circ \mu & =(\mu \otimes \mu) \circ\left(\operatorname{Id}_{H} \otimes c_{H, H} \otimes \operatorname{Id}_{H}\right) \circ(\Delta \otimes \Delta): H \otimes H \rightarrow H \otimes H \\
\Delta \circ \nu & =\nu \otimes \nu: \mathbf{I} \rightarrow H \otimes H \\
\varepsilon \circ \mu & =\varepsilon \otimes \varepsilon: H \otimes H \rightarrow \mathbf{I} \\
\varepsilon \circ \nu & =\operatorname{Id}_{\mathbf{I}}: \mathbf{I} \rightarrow \mathbf{I}
\end{aligned}
$$

A bialgebra morphism is a morphism which respects UAA and coUAA structures simultaneously.

$\rightarrow$ If moreover $H$ has an antipode, i.e., a morphism $s: H \rightarrow H$ satisfying

$$
\mu \circ\left(s \otimes \operatorname{Id}_{H}\right) \circ \Delta=\mu \circ\left(\operatorname{Id}_{H} \otimes s\right) \circ \Delta=\nu \circ \varepsilon
$$

then it is called a Hopf algebra in $\mathcal{C}$.

The notions of (braided) algebra and coalgebra, and of module and comodule, are mutually dual, while that of braiding, of bialgebra and Hopf algebra are self-dual; see [7,15] for more details on the categorical duality. Graphically, applying this duality consists simply in turning all the diagrams upside down, i.e., taking a horizontal mirror image. For instance, Figure 2 contains the graphical depictions of the associativity and the coassociativity axioms. Here and afterwards a multiplication $\mu$ is represented as $\lambda$, and a comultiplication $\Delta-$ as $\%$.

Figure 2. Associativity and coassociativity.

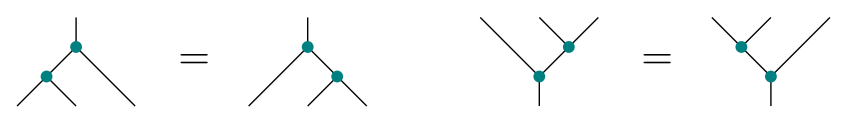

Graphical versions of several other axioms from the above definition are presented on Figures 3 and 4.

Figure 3. Compatibility conditions for a braiding and a comultiplication.

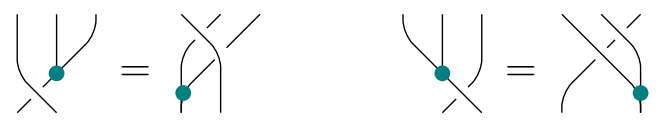

Figure 4. Main bialgebra axiom (15).

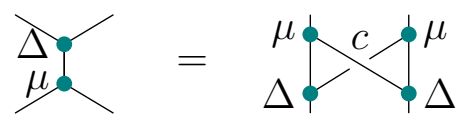

Note that (15) is the only bialgebra axiom requiring a braiding on the underlying category $\mathcal{C}$. 


\subsection{R-Matrices}

From now on we work in a symmetric category $(\mathcal{C}, \otimes, \mathbf{I}, c)$. Fix a bialgebra $H$ in $\mathcal{C}$. A bialgebra structure on $H$ is precisely what is needed for the category ${ }_{H} \mathrm{Mod}$ of its left modules to be monoidal: the tensor product $M \otimes N$ of $H$-modules $\left(M, \lambda_{M}\right)$ and $\left(N, \lambda_{N}\right)$ is endowed with the $H$-module structure

$$
\lambda_{M \otimes N}:=\left(\lambda_{M} \otimes \lambda_{N}\right) \circ\left(\operatorname{Id}_{H} \otimes c_{H, M} \otimes \operatorname{Id}_{N}\right) \circ\left(\Delta \otimes \operatorname{Id}_{M \otimes N}\right)
$$

and the unit object $\mathbf{I}$ is endowed with the $H$-module structure

$$
\lambda_{\mathbf{I}}:=\varepsilon: H \otimes \mathbf{I}=H \rightarrow \mathbf{I}
$$

In what follows we always assume this monoidal structure on ${ }_{H} \mathrm{Mod}$.

If one wants the category ${ }_{H}$ Mod to be braided (and thus to provide solutions to the Yang-Baxter equation), an additional quasi-triangular structure should be imposed on $H$. The growing interest in quasi-triangular structures can thus be partially explained by their capacity to produce highly non-trivial solutions to the YBE. The most famous example is given by quantum groups (see for instance [16]), which will not be discussed here.

Definition 2.6. A bialgebra $H$ in $\mathcal{C}$ is called quasi-triangular if it is endowed with an $R$-matrix, i.e., a morphism $R: \mathbf{I} \rightarrow H \otimes H$ satisfying the following conditions:

1. $\left(\operatorname{Id}_{H} \otimes \Delta\right) \circ R=\left(\mu^{o p} \otimes \operatorname{Id}_{H \otimes H}\right) \circ c^{2} \circ(R \otimes R)$,

2. $\left(\Delta \otimes \operatorname{Id}_{H}\right) \circ R=\left(\operatorname{Id}_{H \otimes H} \otimes \mu\right) \circ c^{2} \circ(R \otimes R)$,

3. $\mu_{H \otimes H} \circ(R \otimes \Delta)=\mu_{H \otimes H} \circ\left(\Delta^{o p} \otimes R\right)$,

where $c^{2}$ is a shorthand notation for $\operatorname{Id}_{H} \otimes c_{H, H} \otimes \operatorname{Id}_{H}$,

$$
\mu_{H \otimes H}:=(\mu \otimes \mu) \circ c^{2}:(H \otimes H) \otimes(H \otimes H) \rightarrow H \otimes H
$$

is the standard multiplication on the tensor product of two UAAs, and

$$
\mu^{o p}:=\mu \circ c_{H, H}, \quad \Delta^{o p}:=c_{H, H} \circ \Delta
$$

are the twisted multiplication and comultiplication respectively.

The R-matrix $R$ is called invertible if there exists a morphism $R^{-1}: \mathbf{I} \rightarrow H \otimes H$ such that

$$
\mu_{H \otimes H} \circ\left(R \otimes R^{-1}\right)=\mu_{H \otimes H} \circ\left(R^{-1} \otimes R\right)=\nu \otimes \nu
$$

Figure 5 shows a graphical version of the conditions from the definition.

Figure 5. Axioms for an R-matrix.
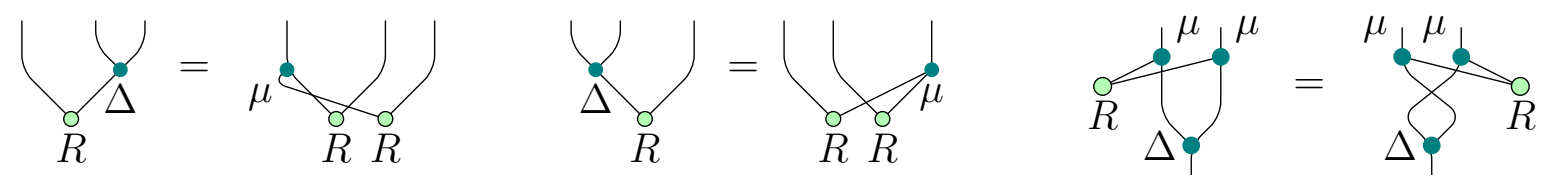

A well-known result affirms that a quasi-triangular bialgebra structure on $H$ is precisely what is needed for its module category to be braided: 
Theorem 1. The category ${ }_{H} \mathrm{Mod}$ of left modules over a quasi-triangular bialgebra $H$ in $\mathcal{C}$ can be endowed with the following braiding (cf. Figure 6):

$$
c_{M, N}^{R}:=c_{M, N} \circ\left(\lambda_{M} \otimes \lambda_{N}\right) \circ\left(\operatorname{Id}_{H} \otimes c_{H, M} \otimes \operatorname{Id}_{N}\right) \circ\left(R \otimes \operatorname{Id}_{M \otimes N}\right)
$$

Here $R$ is the $R$-matrix of $H$, and $c$ is the underlying symmetric braiding of the category $\mathcal{C}$.

If the R-matrix is moreover invertible, then the braiding $c^{R}$ is invertible as well, with

$$
\left(c_{M, N}^{R}\right)^{-1}:=\left(\lambda_{M} \otimes \lambda_{N}\right) \circ\left(\operatorname{Id}_{H} \otimes c_{H, M} \otimes \operatorname{Id}_{N}\right) \circ\left(R^{-1} \otimes c_{N, M}\right)
$$

Figure 6. A braiding for $H$-modules.

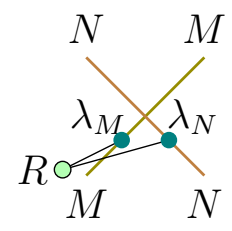

All the statements of the theorem can be verified directly (see [16] or any other book on quantum groups). In Section 2.4 we will see an indirect proof based on a Yetter-Drinfel'd module interpretation of modules over a quasi-triangular bialgebra.

If one is only interested in solutions to the YBE, the following corollary of the above theorem is sufficient:

Corollary 2.7. Given a quasi-triangular bialgebra $(H, R)$ in $\mathcal{C}$, any left module $M$ over $H$ is a braided object in $\mathcal{C}$, with the braiding $\sigma_{M}=c_{M, M}^{R}$. This braiding is invertible if the $R$-matrix $R$ is.

Proof. Apply Lemma 2.4 to the braided category structure from Theorem 1.

\subsection{Yetter-Drinfel'd Modules}

Yetter-Drinfel'd modules are known to be at the origin of a very vast family of solutions to the Yang-Baxter equation. According to [17-19], this family is complete if one restricts oneself to finite-dimensional solutions over a field $\mathbb{k}$. This led L.A. Lambe and D.E. Radford to use the eloquent term quantum Yang-Baxter module instead of the more historical term Yetter-Drinfel'd module, cf. [12]. We recall here the definition of this structure and its most important properties.

Definition 2.8. A Yetter-Drinfel'd $(=Y D)$ module structure over a bialgebra $H$ in a symmetric category $\mathcal{C}$ consists of a left $H$-module structure $\lambda$ and a right $H$-comodule structure $\delta$ on an object $M$, satisfying the Yetter-Drinfel'd compatibility condition (cf. Figure 7)

$$
\begin{gathered}
\left(\operatorname{Id}_{M} \otimes \mu\right) \circ\left(\delta \otimes \operatorname{Id}_{H}\right) \circ c_{H, M} \circ\left(\operatorname{Id}_{H} \otimes \lambda\right) \circ\left(\Delta \otimes \operatorname{Id}_{M}\right)= \\
(\lambda \otimes \mu) \circ\left(\operatorname{Id}_{H} \otimes c_{H, M} \otimes \operatorname{Id}_{H}\right) \circ(\Delta \otimes \delta)
\end{gathered}
$$

The category of YD modules over a bialgebra $H$ (with, as morphisms, those which are simultaneously $H$-module and $H$-comodule morphisms) is denoted by ${ }_{H} \mathbf{Y D}^{H}$. 
Figure 7. Yetter-Drinfel'd compatibility condition.

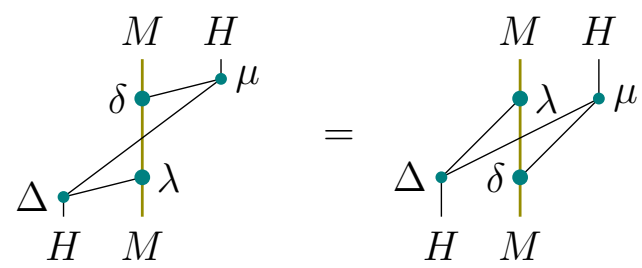

The name left-right Yetter-Drinfel'd module is more appropriate for the structure described in the definition; we shorten it for simplicity. One can also define right-left and (in the case when $H$ is a Hopf algebra) right-right and left-left YD modules. If the antipode $s$ of $H$ is invertible, then all these notions are equivalent due to the notable correspondence between left and right $H$-module structures (similarly for comodules). For example, a right action can be transformed to a left one via

$$
\lambda:=\rho \circ\left(\operatorname{Id}_{M} \otimes s^{-1}\right) \circ c_{H, M}: H \otimes M \rightarrow M=\rho \oint s^{-1}
$$

Example 2.9. A simple but sufficiently insightful example is given by the group algebra $H=\mathbb{k} G$ of a finite group $G$, which is a Hopf algebra via the linearization of the maps $\Delta(g)=g \otimes g, \varepsilon(g)=1$ and $s(g)=g^{-1}$ for all $g \in G$. For such an $H$, the notion of left $H$-module (in the category $\mathbf{V e c t}_{\mathbb{k}}$ ) is easily seen to reduce to that of a $\mathbb{k}$-linear representation of $G$, and the notion of right $H$-comodule to that of a $G$-graded vector space $M=\bigoplus_{g \in G} M_{g}$, with

$$
\delta(m)=m \otimes g \quad \forall m \in M_{g}
$$

The compatibility condition (YD) reads in this setting

$$
g \cdot M_{h} \subseteq M_{g h g^{-1}} \quad \forall g, h \in G
$$

(here the left $H$-action on $M$ is denoted by a dot). In particular, $H$ becomes a YD module over itself when endowed with the $G$-grading $H=\bigoplus_{g \in G} H_{g}, H_{g}:=\mathbb{k} g$ and the adjoint $G$-action

$$
g \cdot h:=g h g^{-1}
$$

The category ${ }_{H} \mathbf{Y D}^{H}$ can be endowed with a monoidal structure in several ways (cf. [12]). We choose here the structure which makes the forgetful functor

$$
\text { For } \begin{array}{r}
{ }_{H} \mathbf{Y D}^{H}{ }{ }_{H} \mathbf{M o d} \\
(M, \lambda, \delta) \longmapsto(M, \lambda)
\end{array}
$$

monoidal, where ${ }_{H}$ Mod is endowed with the monoidal structure described in Section 2.2. Concretely, the tensor product $M \otimes N$ of YD modules $\left(M, \lambda_{M}, \delta_{M}\right)$ and $\left(N, \lambda_{N}, \delta_{N}\right)$ is endowed with the $H$-module structure (16) and the $H$-comodule structure

$$
\delta_{M \otimes N}:=\left(\operatorname{Id}_{M \otimes N} \otimes \mu^{o p}\right) \circ\left(\operatorname{Id}_{M} \otimes c_{H, N} \otimes \operatorname{Id}_{H}\right) \circ\left(\delta_{M} \otimes \delta_{N}\right)
$$

and the unit object $\mathbf{I}$ is endowed with the $H$-modules structure (17) and the $H$-comodule structure

$$
\delta_{\mathbf{I}}:=\nu: \mathbf{I} \rightarrow H=\mathbf{I} \otimes H
$$


Note that using the twisted multiplication $\mu^{o p}$ in the definition of $\delta_{M \otimes N}$ is essential for assuring its YD compatibility with $\lambda_{M \otimes N}$.

The monoidal category ${ }_{H} \mathbf{Y D}^{H}$ defined this way possesses a famous braided structure:

Theorem 2. The category ${ }_{H} \mathrm{YD}^{H}$ of left-right Yetter-Drinfel'd modules can be endowed with the following braiding (cf. Figure 8):

$$
c_{M, N}^{Y D}:=\left(\operatorname{Id}_{N} \otimes \lambda_{M}\right) \circ\left(\delta_{N} \otimes \operatorname{Id}_{M}\right) \circ c_{M, N}
$$

If $H$ is moreover a Hopf algebra with the antipode s, then the braiding $c^{Y D}$ is invertible, with

$$
\left(c_{M, N}^{Y D}\right)^{-1}:=c_{N, M} \circ\left(\operatorname{Id}_{N} \otimes \lambda_{M}\right) \circ\left(\operatorname{Id}_{N} \otimes s \otimes \operatorname{Id}_{M}\right) \circ\left(\delta_{N} \otimes \operatorname{Id}_{M}\right)
$$

Figure 8. A braiding for left-right YD modules.

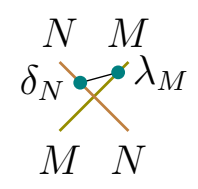

The theorem can be proved by an easy direct verification.

Remark 2.10. The category ${ }_{H} \mathbf{Y D}^{H}$ can be endowed with a monoidal structure alternative to Equations (16) and (22). Namely, one can endow the tensor product of YD modules $M$ and $N$ with the "twisted" module and usual comodule structure:

$$
\begin{aligned}
& \dot{\lambda}_{M \otimes N}:=\left(\lambda_{M} \otimes \lambda_{N}\right) \circ\left(\operatorname{Id}_{H} \otimes c_{H, M} \otimes \operatorname{Id}_{N}\right) \circ\left(\Delta^{o p} \otimes \operatorname{Id}_{M \otimes N}\right) \\
& \stackrel{\delta}{M \otimes N}_{M}:=\left(\operatorname{Id}_{M \otimes N} \otimes \mu\right) \circ\left(\operatorname{Id}_{M} \otimes c_{H, N} \otimes \operatorname{Id}_{H}\right) \circ\left(\delta_{M} \otimes \delta_{N}\right)
\end{aligned}
$$

Theorem 2 remains true in this setting if one replaces the braiding $c^{Y D}$ with its alternative version

$$
\stackrel{\circ}{M, N}_{M D}^{Y D}:=c_{M, N} \circ\left(\operatorname{Id}_{M} \otimes \lambda_{N}\right) \circ\left(\delta_{M} \otimes \operatorname{Id}_{N}\right)
$$

This construction is best explained graphically. First, observe that the notions of bialgebra, YD module and braiding are stable by the central symmetry. In other words, the sets of diagrams representing the axioms defining these notions are stable by an angle $\pi$ rotation (hence the notations $\stackrel{\circ}{c}$ etc. evoking rotation). Now, an angle $\pi$ rotation of the $H$-module structure (16) is the $H$-comodule structure (27), and similarly for Equations (22) and (26). To conclude, note that the braiding $c^{Y D}$ is precisely an angle $\pi$ rotation of $c^{Y D}$ (cf. Figures 8 and 9). This alternative structure will be used in Section 3.

Figure 9. An alternative braiding for left-right YD modules.

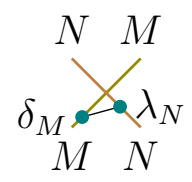


If one is only interested in solutions to the YBE, the following corollary is sufficient:

Corollary 2.11. Given a bialgebra $H$ in $\mathcal{C}$, any left-right $Y D$ module $M$ over $H$ is a braided object in $\mathcal{C}$, with the braiding $\sigma_{M}=c_{M, M}^{Y D}$. This braiding is invertible if $H$ is moreover a Hopf algebra.

Proof. Apply Lemma 2.4 to the braided category structure from Theorem 2.

Example 2.12. Applied to Example 2.9, the corollary gives an invertible braiding

$$
c_{H, H}^{Y D}(h \otimes g)=g \otimes g h g^{-1} \quad \forall g, h \in G
$$

for $H=\mathbb{k} G$. One recognizes (the linearization of) a familiar braiding for groups, which can alternatively be obtained using the machinery of self-distributive structures.

\subsection{A Category Inclusion}

The braided categories constructed in the two previous sections exhibit apparent similarities. We explain them here by interpreting the category ${ }_{H} \mathrm{Mod}$ of left modules over a quasi-triangular bialgebra $H$ in a symmetric category $\mathcal{C}$ as a full braided subcategory of the category ${ }_{H} \mathbf{Y} \mathbf{D}^{H}$ of left-right Yetter-Drinfel'd modules over $H$. We further introduce a weaker notion of R-matrix for which the above category inclusion still holds true, in general without respecting the monoidal structures. In particular, if one is interested only in constructing solutions to the Yang-Baxter equation (cf. Corollaries 2.7 and 2.11), this weaker notion suffices.

Let $(\mu, \nu)$ and $(\Delta, \varepsilon)$ be a UAA and, respectively, a coUAA structures on an object $H$ of $\mathcal{C}$. A preliminary remark is first due.

Remark 2.13. The definition of Yetter-Drinfel'd module actually requires (not necessarily compatible) UAA and coUAA structures on $H$ only. The category ${ }_{H} \mathbf{Y D}^{H}$ is no longer monoidal in this setting. However, a direct verification shows that Corollary 2.11 still holds true (the argument closely repeats that from the proof of Theorem 5 , point 2).

We thus do not suppose $H$ to be a bialgebra unless explicitely specified.

Take a left module $(M, \lambda)$ over the algebra $H$ and a morphism $R: \mathbf{I} \rightarrow H \otimes H$. Put, as in Figure 10,

$$
\delta^{R}:=c_{H, M} \circ\left(\operatorname{Id}_{H} \otimes \lambda\right) \circ\left(R \otimes \operatorname{Id}_{M}\right): M \rightarrow M \otimes H
$$

Figure 10. Module + R-matrix $\longmapsto$ comodule.

$$
\delta^{R}:=\operatorname{lof}_{\lambda}
$$

Now try to determine conditions on $R$ which make $\left(M, \lambda, \delta^{R}\right)$ a left-right Yetter-Drinfel'd module for any $M$. One arrives to the following set of axioms:

Definition 2.14. A morphism $R: \mathbf{I} \rightarrow H \otimes H$ is called a weak $R$-matrix for a UAA and a coUAA object $(H, \mu, \nu, \Delta, \varepsilon)$ in $\mathcal{C}$ if (cf. Figure 11) 
1. $\left(\Delta \otimes \operatorname{Id}_{H}\right) \circ R=\left(\operatorname{Id}_{H \otimes H} \otimes \mu\right) \circ c^{2} \circ(R \otimes R)$,

2. $\left(\varepsilon \otimes \operatorname{Id}_{H}\right) \circ R=\nu$,

3. $\mu_{H \otimes H} \circ(R \otimes \Delta)=\mu_{H \otimes H} \circ\left(\Delta^{o p} \otimes R\right)$.

Figure 11. Axioms for a weak R-matrix.
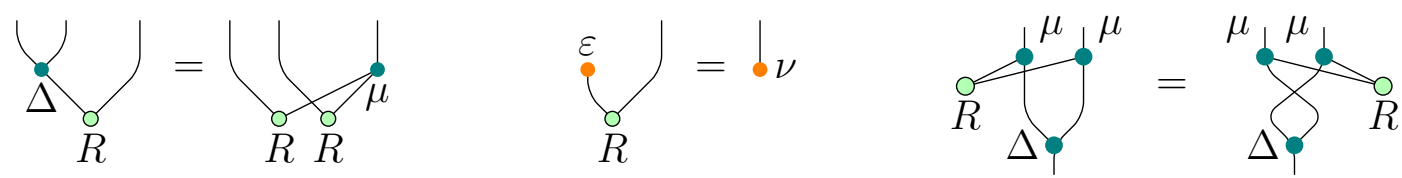

One can informally interpret the first two conditions by saying that $R$ provides a duality between the UAA $(H, \mu, \nu)$ on the right and the coUAA $(H, \Delta, \varepsilon)$ on the left.

Remark 2.15. If the weak R-matrix is invertible, then axiom 2 is a consequence of 1: apply $\operatorname{Id}_{H} \otimes \varepsilon \otimes \operatorname{Id}_{H}$ to both sides, then multiply by $R^{-1}$ on the left and apply $\varepsilon \otimes \operatorname{Id}_{H}$.

We also need the following notion:

Definition 2.16. A strong R-matrix is a weak R-matrix satisfying two additional axioms (Figure 12):

1'. $\left(\operatorname{Id}_{H} \otimes \Delta\right) \circ R=\left(\mu^{o p} \otimes \operatorname{Id}_{H \otimes H}\right) \circ c^{2} \circ(R \otimes R)$,

2'. $\left(\operatorname{Id}_{H} \otimes \varepsilon\right) \circ R=\nu$.

Figure 12. Additional axioms for a strong R-matrix.

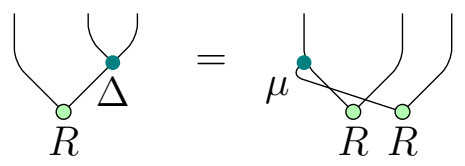

$\int_{0}^{\varepsilon}=1 \nu$

A strong R-matrix satisfies all usual R-matrix axioms by definition. For invertible R-matrices, arguments similar to those from Remark 2.15 show that axiom 2'. is a consequence of 1'., hence the notions of strong and usual R-matrix coincide.

As it was hinted before Definition 2.14, a weak R-matrix for $H$ allows to upgrade a module structure over the algebra $H$ into a Yetter-Drinfel'd module structure:

Theorem 3. Take a UAA and a coUAA object $(H, \mu, \nu, \Delta, \varepsilon)$ in $\mathcal{C}$ equipped with a weak R-matrix $R$.

1. For any left $H$-module $(M, \lambda)$, the data $\left(M, \lambda, \delta^{R}\right)$ form a left-right $Y D$ module over $H$.

2. For any two $H$-modules (and hence YD modules) $\left(M, \lambda_{M}\right)$ and $\left(N, \lambda_{N}\right)$, the morphism $c_{M, N}^{R}$ from Equation (20) coincides with $c_{M, N}^{Y D}$ from Equation (24) and, for $N=M$, defines a braiding for the object $M$ in $\mathcal{C}$.

3. The category ${ }_{H} \mathrm{Mod}$ can be seen as a full subcategory of ${ }_{H} \mathbf{Y D}^{H}$ via the inclusion

$$
\begin{aligned}
i_{R}:{ }_{H} \operatorname{Mod} & \longleftrightarrow{ }_{H} \mathbf{Y D}^{H} \\
(M, \lambda) & \longmapsto\left(M, \lambda, \delta^{R}\right)
\end{aligned}
$$

4. If $H$ is a bialgebra and $R$ is a strong $R$-matrix, then the functor $i_{R}$ is braided monoidal. 
Proof. 1. The first two conditions from the definition of weak R-matrix guarantee that $\delta^{R}$ defines a coUAA comodule, while the last one implies the YD compatibility (YD).

2. The equality of the two morphisms follows from the choice of $\delta^{R}$. The fact that $c_{M, M}^{Y D}$ is a braiding was noticed in Remark 2.13.

3. We have seen in point 1 that $i_{R}$ is well defined on objects. Further, using the definition of $\delta^{R}$ and the naturality of $c$, one checks that a morphism in $\mathcal{C}$ respecting module structures necessarily respects comodule structures defined by $\delta^{R}$. Thus $i_{R}$ is well defined, full and faithful on morphisms.

4. Let us now show that, under the additional conditions of this point, $i_{R}$ respects monoidal structures. Take two $H$-modules $\left(M, \lambda_{M}\right)$ and $\left(N, \lambda_{N}\right)$. The $H$-module structure $\lambda_{M \otimes N}$ on $M \otimes N$ is given by Equation (16). The functor $i_{R}$ transforms it into a YD module over $H$, with as $H$-comodule structure

$$
\begin{aligned}
\delta_{M \otimes N}^{R}=c_{H, M \otimes N} & \circ\left(\operatorname{Id}_{H} \otimes \lambda_{M \otimes N}\right) \circ\left(R \otimes \operatorname{Id}_{M \otimes N}\right) \\
=c_{H, M \otimes N} & \circ\left(\operatorname{Id}_{H} \otimes \lambda_{M} \otimes \lambda_{N}\right) \circ\left(\operatorname{Id}_{H \otimes H} \otimes c_{H, M} \otimes \operatorname{Id}_{N}\right) \\
& \circ\left(\left(\left(\operatorname{Id}_{H} \otimes \Delta\right) \circ R\right) \otimes \operatorname{Id}_{M \otimes N}\right)
\end{aligned}
$$

Now in ${ }_{H} \mathbf{Y D}^{H}$ the tensor product of $\left(M, \lambda_{M}, \delta_{M}^{R}\right)$ and $\left(N, \lambda_{N}, \delta_{N}^{R}\right)$ has an $H$-module structure given by $\lambda_{M \otimes N}$, and an $H$-comodule structure given by Equation (22):

$$
\begin{aligned}
\delta_{M \otimes N}=\left(\operatorname{Id}_{M \otimes N} \otimes \mu^{o p}\right) \circ\left(\operatorname{Id}_{M} \otimes c_{H, N} \otimes \operatorname{Id}_{H}\right) \circ\left(\delta_{M}^{R} \otimes \delta_{N}^{R}\right) \\
=\left(\operatorname{Id}_{M \otimes N} \otimes \mu^{o p}\right) \circ\left(\operatorname{Id}_{M} \otimes c_{H, N} \otimes \operatorname{Id}_{H}\right) \\
\circ\left(c_{H, M} \otimes c_{H, N}\right) \circ\left(\operatorname{Id}_{H} \otimes \lambda_{M} \otimes \operatorname{Id}_{H} \otimes \lambda_{N}\right) \circ\left(R \otimes \operatorname{Id}_{M} \otimes R \otimes \operatorname{Id}_{N}\right) \\
=c_{H, M \otimes N} \circ\left(\operatorname{Id}_{H} \otimes \lambda_{M} \otimes \lambda_{N}\right) \circ\left(\operatorname{Id}_{H \otimes H} \otimes c_{H, M} \otimes \operatorname{Id}_{N}\right) \\
\circ\left(\left(\left(\mu^{o p} \otimes \operatorname{Id}_{H \otimes H}\right) \circ c^{2} \circ(R \otimes R)\right) \otimes \operatorname{Id}_{M \otimes N}\right)
\end{aligned}
$$

The reader is advised to draw diagrams in order to better follow these calculations. Now, axiom 1'. from the definition of a strong R-matrix is precisely what is needed for the two YD structures on $M \otimes N$ to coincide.

A similar comparison of the standard $H$-comodule structure on the unit object $\mathbf{I}$ of $\mathcal{C}$ with the one induced by $R$ shows that they coincide if and only if axiom 2'. is verified.

Point 2 shows that $i_{R}$ also respects braidings, allowing one to conclude.

Note that in Point 2, $c_{M, N}^{R}=c_{M, N}^{Y D}$ is a morphism in $\mathcal{C}$ and not in ${ }_{H} \mathrm{Mod}$ in general, since the $H$-module structure on $M \otimes N$ is not even defined if $H$ is not a bialgebra.

In the proof of the theorem one clearly sees that the full set of strong R-matrix axioms is necessary only if one wants to construct braided monoidal categories, while the notion of weak R-matrix suffices if one is interested in the "local" structure of objects in $\mathcal{C}$ (in particular, in solutions to the YBE) only.

The relations between different structures from the above theorems can be presented in the following charts (in each of them one starts with a UAA and a coUAA $H$ and a morphism $R: \mathbf{I} \rightarrow H \otimes H$ ): 
1. $H$ is a bialgebra, $R$ is a strong $\mathrm{R}$-matrix

\begin{tabular}{|c|c|c|c|c|}
\hline \multicolumn{2}{|r|}{ Thm 1} & \multirow{2}{*}{\multicolumn{2}{|c|}{$\begin{array}{c}c^{R} \text { is a braiding } \\
\text { on }{ }_{H} \text { Mod }\end{array}$}} & $\operatorname{Crl} 2.7 c_{M . M}^{R}$ is a b \\
\hline $\mathrm{Thm}$ & Thm 2 & & & for $M$ in \\
\hline \multicolumn{2}{|c|}{$\begin{array}{l}{ }_{H} \mathbf{M} \text { od is a full monoidal } \\
\text { subcategory of }{ }_{H} \mathbf{Y D}^{H}\end{array}$} & \multirow[b]{2}{*}{ Rmk 2.13} & & \\
\hline$n 3$ & $\begin{array}{c}{ }_{H} \text { Mod is a full } \\
\text { subcategory of }{ }_{H} \mathbf{Y D}^{H}\end{array}$ & & \multicolumn{2}{|c|}{$\begin{array}{c}c_{M, M}^{R}=c_{M, M}^{Y D} \text { is } \\
\text { a braiding for } M \text { in } \mathcal{C}\end{array}$} \\
\hline
\end{tabular}

Observe that if one disregards all the structural issues and limits oneself to the search of solutions to the YBE, one obtains that the only condition that should be imposed on $R$ so that Equation (20) becomes a braiding is the following:

$$
R_{23} * R_{13} * R_{12}=R_{12} * R_{13} * R_{23}: \mathbf{I} \rightarrow H \otimes H \otimes H
$$

where $R_{12}:=R \otimes \nu, R_{23}:=\nu \otimes R, R_{13}:=\left(\operatorname{Id}_{H} \otimes c_{H, H}\right) \circ R_{12}$, and $*$ stands for the multiplication on $H \otimes H \otimes H$ defined by a formula analogous to Equation (18). This relation is sometimes called algebraic, or quantum, Yang-Baxter equation. Other authors however reserve this term for (YBE).

We finish by showing that in the Hopf algebra case, which is the most common in literature, the invertibility of a weak R-matrix is automatic:

Proposition 2.17. If a Hopf algebra $H$ with the antipode $s$ is endowed with a weak $R$-matrix $R$, then

$$
R^{-1}:=\left(s \otimes \operatorname{Id}_{H}\right) \circ R
$$

defines an inverse for $R$, in the sense of Equation (19).

Proof. Apply $\left(\mu \otimes \operatorname{Id}_{H}\right) \circ\left(s \otimes \operatorname{Id}_{H} \otimes \operatorname{Id}_{H}\right)$, or $\left(\mu \otimes \operatorname{Id}_{H}\right) \circ\left(\operatorname{Id}_{H} \otimes s \otimes \operatorname{Id}_{H}\right)$, to both sides of the axiom 1 from the definition of weak R-matrix. Axiom 2 and the definition (s) of the antipode allow one to conclude.

\section{Yetter-Drinfel'd Modules and the Yang-Baxter Equation: The Story Continued}

\subsection{Braided Systems}

In order to describe further connections between Yetter-Drinfel'd modules and the Yang-Baxter equation, the following notion from [8] will be useful:

Definition 3.1. $\rightarrow$ A braided system in a monoidal category $\mathcal{C}$ is an ordered finite family $V_{1}, V_{2}, \ldots, V_{r}$ of objects in $\mathcal{C}$ endowed with a braiding, i.e., morphisms $\sigma_{i, j}: V_{i} \otimes V_{j} \rightarrow V_{j} \otimes V_{i}$ for $1 \leqslant i \leqslant j \leqslant r$ satisfying the colored Yang-Baxter equation (= cYBE)

$$
\left(\sigma_{j, k} \otimes \operatorname{Id}_{i}\right) \circ\left(\operatorname{Id}_{j} \otimes \sigma_{i, k}\right) \circ\left(\sigma_{i, j} \otimes \operatorname{Id}_{k}\right)=\left(\operatorname{Id}_{k} \otimes \sigma_{i, j}\right) \circ\left(\sigma_{i, k} \otimes \operatorname{Id}_{j}\right) \circ\left(\operatorname{Id}_{i} \otimes \sigma_{j, k}\right)
$$

on all the tensor products $V_{i} \otimes V_{j} \otimes V_{k}$ with $1 \leqslant i \leqslant j \leqslant k \leqslant r$. (Here $\operatorname{Id}_{t}$ stands for $\operatorname{Id}_{V_{t}}$, $1 \leqslant t \leqslant r$.) Such a system is denoted by $\left(\left(V_{i}\right)_{1 \leqslant i \leqslant r} ;\left(\overline{\left.\sigma_{i, j}\right)_{1 \leqslant i \leqslant j \leqslant r}}\right)\right.$ or briefly $(\bar{V}, \bar{\sigma})$.

$\rightarrow$ The rank of a braided system is the number $r$ of its components. 
$\rightarrow$ A braided morphism $\bar{f}:(\bar{V}, \bar{\sigma}) \rightarrow(\bar{W}, \bar{\xi})$ between two braided systems in $\mathcal{C}$ of the same rank $r$ is a collection of morphisms $\left(f_{i} \in \operatorname{Hom}_{\mathcal{C}}\left(V_{i}, W_{i}\right)\right)_{1 \leqslant i \leqslant r}$ respecting the braiding, i.e.,

$$
\left(f_{j} \otimes f_{i}\right) \circ \sigma_{i, j}=\xi_{i, j} \circ\left(f_{i} \otimes f_{j}\right) \quad \forall 1 \leqslant \underline{i \leqslant j} \leqslant r
$$

$\rightarrow$ The category of rank $r$ braided systems and braided morphisms in $\mathcal{C}$ is denoted by $\operatorname{BrSyst}_{r}(\mathcal{C})$.

This notion is a partial generalization of that of braided object in $\mathcal{C}$, in the sense that a braiding is defined only on certain couples of objects (which is underlined in the definition).

Graphically, the $\sigma_{i, j}$ component of a braiding is depicted on Figure 13. According to the definition, one allows a strand to overcross only the strands colored with a smaller or equal index $i \in\{1,2, \ldots, r\}$.

Figure 13. A braiding component.

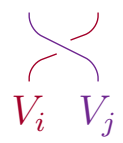

Observe that each component of a braided system is a braided object in $\mathcal{C}$.

Various examples of braided systems coming from algebraic considerations are presented in [8]. Different aspects of those systems are studied in detail there, including their representation and homology theories, generalizing usual representation and homology theories for basic algebraic structures.

In the next section we will describe a rank 3 braided system constructed from a Yetter-Drinfel'd module over a finite-dimensional $\mathbb{k}$-linear bialgebra. The braiding on this system captures all axioms of the YD module structure. Several components of this braiding are inspired by the braiding ${ }^{\circ} Y D$ from Equation (28).

\subsection{Yetter-Drinfel'd Module $\longmapsto$ Braided System}

In this section we work in the category Vect $_{\mathbb{k}}$ of $\mathbb{k}$-vector spaces and $\mathbb{k}$-linear maps, endowed with the usual tensor product over $\mathbb{k}$, with the unit $\mathbb{k}$ and with the flip

$$
c(v \otimes w)=w \otimes v \quad \forall v \in V, w \in W
$$

as symmetric braiding. Note however that one could stay in the general setting of a symmetric category and replace the property "finite-dimensional" with "admitting a dual" in what follows.

Recall the classical Sweedler's notation without summation sign for comultiplications and coactions in Vect $_{\mathbb{k}}$, used here and further in the paper:

$$
\begin{array}{lrl}
\Delta(h):=h_{(1)} \otimes h_{(2)} \in H \otimes H & \forall h \in H \\
\delta(m):=m_{(0)} \otimes m_{(1)} \in M \otimes H & \forall m \in M
\end{array}
$$

We now describe by giving explicit formulas a braided system constructed from an arbitrary YD module. In Section 3.4 this construction will be explained from a more conceptual viewpoint. 
Theorem 4. Let $H$ be a finite-dimensional $\mathbb{k}$-linear bialgebra, and let $M$ be a Yetter-Drinfel'd module over $H$. Then the rank 3 system $\left(H, M, H^{*}\right)$ can be endowed with the following braiding:

$$
\begin{array}{rlrl}
\sigma_{H, H}: h_{1} \otimes h_{2} \mapsto h_{1} h_{2} \otimes 1, & \sigma_{M, M}: m_{1} \otimes m_{2} \mapsto m_{1} \otimes m_{2} \\
\sigma_{H^{*}, H^{*}}: l_{1} \otimes l_{2} \mapsto \varepsilon \otimes l_{1} l_{2}, & \sigma_{H, M}: h \otimes m & \mapsto h_{(2)} m \otimes h_{(1)} \\
\sigma_{H, H^{*}}: h \otimes l \mapsto l_{(1)}\left(h_{(2)}\right) l_{(2)} \otimes h_{(1)}, & \sigma_{M, H^{*}}: m \otimes l & \mapsto l_{(1)}\left(m_{(1)}\right) l_{(2)} \otimes m_{(0)}
\end{array}
$$

Here the signs for multiplication morphisms in $H$ and $H^{*}$, as well as for the $H$-action on $M$, are omitted for simplicity. Further, $\mathbf{1}$ denotes the unit of $H$, i.e., $\nu(\alpha)=\alpha \mathbf{1}$ for all $\alpha \in \mathbb{k}$.

Multiplication, comultiplication and other structures on $H^{*}$ used in the theorem are obtained from those on $H$ by duality. For instance,

$$
\begin{aligned}
l_{1} l_{2}(h):=\Delta^{*}\left(l_{1} \otimes l_{2}\right)(h):=l_{1}\left(h_{(2)}\right) l_{2}\left(h_{(1)}\right) & \forall l_{1}, l_{2} \in H^{*}, h \in H \\
\mathbf{1}_{H^{*}}(h):=\left(\varepsilon_{H}\right)^{*}(1)(h):=\varepsilon_{H}(h) & \forall h \in H
\end{aligned}
$$

See [8] for some comments on an alternative definition of the duality between $H \otimes H$ and $H^{*} \otimes H^{*}$, which gives a slightly different formula for $\Delta^{*}$, more common in literature.

The multiplication $\Delta^{*}$ on $H^{*}$ is graphically depicted on Figure 14 . Here and afterwards dashed lines stand for $H^{*}$, and $e v$ denotes one of the evaluation maps

$$
\begin{aligned}
& \text { ev }: \quad H^{*} \otimes H \longrightarrow \mathbb{k} \text {, } \\
& \text { or } \\
& H \otimes H^{*} \longrightarrow \mathbb{k}, \\
& l \otimes h \longmapsto l(h) ; \\
& h \otimes l \longmapsto l(h) .
\end{aligned}
$$

Figure 14. Dual structures on $H^{*}$ via the "rainbow" duality.

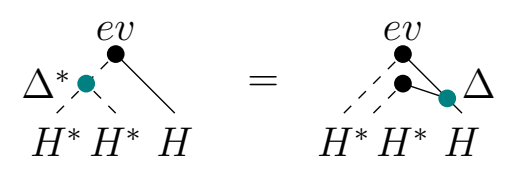

All components of the braiding from the theorem are presented on Figure 15.

Figure 15. A braiding for the system $\left(H, M, H^{*}\right)$.

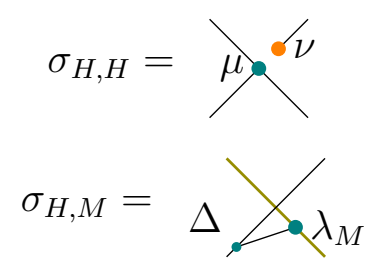

$$
\begin{gathered}
\sigma_{H, H^{*}}= \\
\sigma_{M, M}=\mid
\end{gathered}
$$

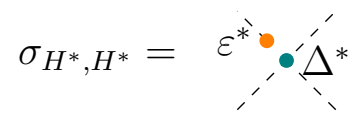$$
\sigma_{M, H^{*}}=\delta_{M}
$$

In order to prove the theorem, one can simply verify by tedious but straightforward computations the $\left(\begin{array}{c}3+2 \\ 2\end{array}\right)=10$ instances of the cYBE involved in the definition of rank 3 braided system. A more conceptual proof will be given in the more general setting of Sections 3.3 and 3.4 (cf. Example 3.11). In subsequent sections we will also study functoriality, precision and homology questions for this braided system. 


\subsection{Yetter-Drinfel'd Systems: Definition and Braided Structure}

We introduce here the notion of Yetter-Drinfel'd system in a symmetric category $\mathcal{C}$ and show how to endow it with a braiding. The braided system from Section 3.2 turns out to be a particular case of this general construction. In Section 3.4 we will consider other particular cases, namely a braided system encoding the bialgebra structure (cf. [8]) and a braided system of several Yetter-Drinfel'd modules over a common bialgebra $H$. The latter will lead to a "braided" interpretation of the monoidal structures on the category ${ }_{H} \mathbf{Y D}^{H}$ (Section 3.6).

Let an object $H$ in $\mathcal{C}$ be endowed with a UAA structure $\left(\mu_{H}, \nu_{H}\right)$ and a coUAA structure $\left(\Delta_{H}, \varepsilon_{H}\right)$, a priori not compatible.

Definition 3.2. $\rightarrow$ A (left-right) Yetter-Drinfel'd module algebra over $H$ is the datum of a UAA structure $(\mu, \nu)$ and a YD structure $(\lambda, \delta)$ on an object $V$ in $\mathcal{C}$, such that $\mu$ and $\nu$ are morphisms of YD modules (see Equations (26), (27) and (17), (23) for the $H$-(co)module structure of $V \otimes V$ and of I):

$$
\begin{aligned}
\delta \circ \mu & =\left(\mu \otimes \mu_{H}\right) \circ\left(\operatorname{Id}_{V} \otimes c_{H, V} \otimes \operatorname{Id}_{H}\right) \circ(\delta \otimes \delta) \\
\lambda \circ\left(\operatorname{Id}_{H} \otimes \mu\right) & =\mu \circ(\lambda \otimes \lambda) \circ\left(\operatorname{Id}_{H} \otimes c_{H, V} \otimes \operatorname{Id}_{V}\right) \circ\left(\Delta_{H}^{o p} \otimes \operatorname{Id}_{V \otimes V}\right) \\
\delta \circ \nu & =\nu \otimes \nu_{H} \\
\lambda \circ\left(\operatorname{Id}_{H} \otimes \nu\right) & =\varepsilon_{H} \otimes \nu
\end{aligned}
$$

$\rightarrow$ The category of YD module algebras over $H$ in $\mathcal{C}$ (with as morphisms those which are simultaneously UAA and YD module morphisms) is denoted by ${ }_{H} \mathrm{YDAlg}^{H}$.

$\rightarrow$ Omitting the comodule structure $\delta$ from the definition, one gets the notion of $H$-module algebra. $H$-comodule algebras are defined similarly.

The name $H^{c o p}$-module algebra would be more appropriate than $H$-module algebra since we use the twisted compatibility condition (36). However we opt for the simpler notation since it causes no confusion in what follows.

For the reader's convenience, we give in Figure 16 a graphical form of the compatibility relations (35)-(38). Here and afterwards we use thick colored lines for the module $V$, and thin black lines for $H$.

Figure 16. Compatibilities between UAA and YD structures.
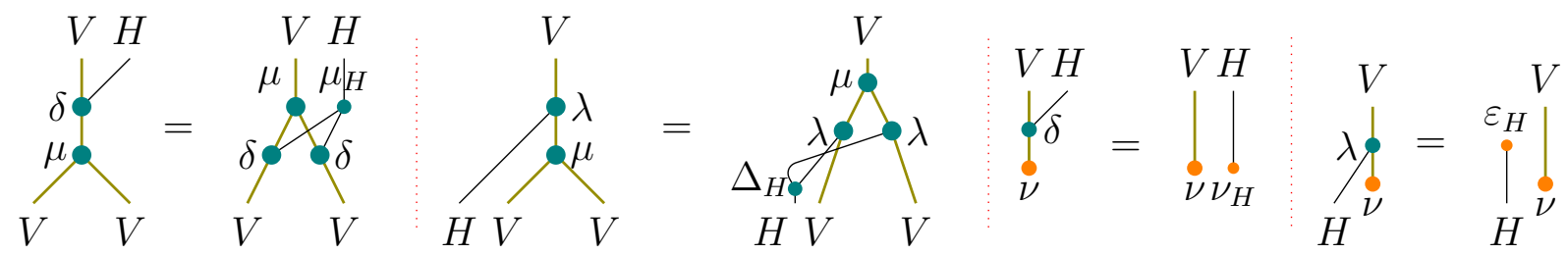

Definition 3.3. A (left-right) Yetter-Drinfel'd system over $H$ ( $=H$-YD system) in $\mathcal{C}$ is an ordered finite family $V_{1}, V_{2}, \ldots, V_{r}$ of objects endowed with the following structure:

1. $\left(V_{1}, \mu_{1}, \nu_{1}, \delta_{1}\right)$ is an $H$-comodule algebra; 
2. $\left(V_{r}, \mu_{r}, \nu_{r}, \lambda_{r}\right)$ is an $H$-module algebra;

3. $\left(V_{i}, \mu_{i}, \nu_{i}, \lambda_{i}, \delta_{i}\right)$ is a YD module algebra over $H$ for all $1<i<r$.

Now we show how to endow a YD system with a braiding:

Theorem 5. A braiding can be defined on a Yetter-Drinfel'd system $\left(V_{1}, \ldots V_{r}\right)$ over $H$ by

$$
\begin{aligned}
\sigma_{i, i} & :=\nu_{i} \otimes \mu_{i} \text { or } \mu_{i} \otimes \nu_{i}: V_{i} \otimes V_{i} \rightarrow V_{i} \otimes V_{i}, \\
\sigma_{i, j} & :=c_{V_{i}, V_{j}}^{Y}=c_{V_{i}, V_{j}} \circ\left(\operatorname{Id}_{V_{i}} \otimes \lambda_{j}\right) \circ\left(\delta_{i} \otimes \operatorname{Id}_{V_{j}}\right): V_{i} \otimes V_{j} \rightarrow V_{j} \otimes V_{i}, i<j .
\end{aligned}
$$

The $\sigma_{i, j}$ components of this braiding with $i<j$ are invertible if $H$ is a Hopf algebra.

In order to prove the theorem, the following result from [8] will be useful:

Proposition 3.4. Take r UAAs $\left(V_{i}, \mu_{i}, \nu_{i}\right)_{1 \leqslant i \leqslant r}$ in a monoidal category $\mathcal{C}$ and, for each couple of subscripts $1 \leqslant i<j \leqslant r$, take a morphism $\xi_{i, j}: V_{i} \otimes V_{j} \rightarrow V_{j} \otimes V_{i}$ natural with respect to $\nu_{i}$ and $\nu_{j}$ (in the sense of a multi-object version of Equations (10) and (11)). The following statements are then equivalent:

1. The morphisms

$$
\xi_{i, i}:=\nu_{i} \otimes \mu_{i}
$$

complete the $\xi_{i, j}$ 's into a braided system structure on $\bar{V}$.

2. Each $\xi_{i, j}$ is natural with respect to $\mu_{i}$ and $\mu_{j}$ (in the sense of a multi-object version of Equations (8) and (9)) and, for each triple $i<j<k$, the $\xi_{i, j}$ 's satisfy the colored Yang-Baxter equation on $V_{i} \otimes V_{j} \otimes V_{k}$.

We call braided systems described in the proposition braided systems of UAAs. In [8] this structure was shown to be equivalent to that of a multi-braided tensor product of UAAs.

The associativity braidings $\xi_{i, i}$ from Equation (39) were introduced in [13], where they were shown to encode the associativity (in the sense that the YBE for $\xi_{i, i}$ is equivalent to $\mu_{i}$ being associative, if one imposes that $\nu_{i}$ is a unit for $\mu_{i}$ ) and to capture many structural properties of the latter. We denote them by $\sigma_{A s s}\left(V_{i}\right)$. Observe that they are highly non-invertible in general.

Remark 3.5. Some or all of the morphisms $\xi_{i, i}$ in the proposition can be replaced with their right versions

$$
\sigma_{\text {Ass }}^{r}\left(V_{i}\right):=\mu_{i} \otimes \nu_{i}
$$

Proof of the theorem. First notice that the compatibility conditions (37) and (38) between the units $\nu_{i}$ of the $V_{i}$ 's and the $H$-(co)module structures on the $V_{i}$ 's (cf. two last pictures on Figure 16) ensure that $c_{V_{i}, V_{j}}^{\circ D}$ is natural with respect to units. In order to deduce the theorem from Proposition 3.4, it remains to check the following two conditions:

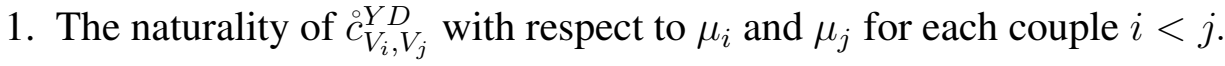

Figure 17 contains a graphical proof of the naturality with respect to $\mu_{i}$, the case of $\mu_{j}$ being similar. Labels $V_{i}, V_{j}, H, \mu_{i}$ etc. are omitted for compactness. 
Figure 17. Naturality with respect to $\mu_{i}$ : a graphical proof.

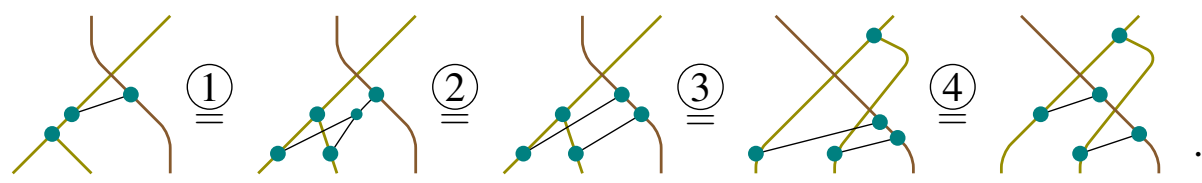

Here we use:

(1) the compatibility (35) between $\delta_{i}$ and $\mu_{i}$ (cf. Figure 16, picture 1);

(2) the definition of $H$-module for $V_{j}$;

(3) the naturality of the symmetric braiding $c$ of $\mathcal{C}$;

(4) the naturality and the symmetry (5) of $c$.

2. Condition (cYBE) on $V_{i} \otimes V_{j} \otimes V_{k}$ for each triple $i<j<k$.

Due to the naturality of $c$, this condition is equivalent to the one graphically presented on Figure 18.

Figure 18. Yang-Baxter equation for Yetter-Drinfel'd modules.

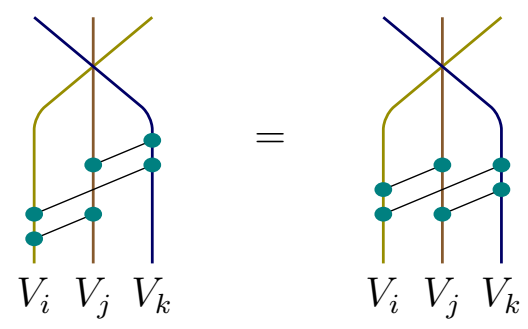

To prove this, one needs

$\rightarrow$ the defining property of right $H$-comodule for $V_{i}$,

$\rightarrow$ the defining property of left $H$-module for $V_{k}$,

$\rightarrow$ the Yetter-Drinfel'd property for $V_{j}$.

As for the invertibility statement, if $H$ is a Hopf algebra, then the inverse of $\sigma_{i, j}={ }_{c}^{\circ}{ }_{V_{i}, V_{j}}^{Y D}, i<j$, can be explicitely given by the formula

$$
\sigma_{i, j}^{-1}=\left(\operatorname{Id}_{V_{i}} \otimes \lambda_{j}\right) \circ\left(\operatorname{Id}_{V_{i}} \otimes s \otimes \operatorname{Id}_{V_{j}}\right) \circ\left(\delta_{i} \otimes \operatorname{Id}_{V_{j}}\right) \circ c_{V_{j}, V_{i}}: V_{j} \otimes V_{i} \rightarrow V_{i} \otimes V_{j}
$$

Remark 3.6. No compatibility between the algebra and coalgebra structures on $H$ are demanded explicitly. However, other properties of a YD system dictate that $H$ should be not too far from a bialgebra, at least as far as (co)actions are concerned. This statement is made concrete and is proved (in the $H$-comodule case) in Figure 19; cf. Figure 4 for the definition of bialgebra.

Figure 19. Almost a bialgebra.

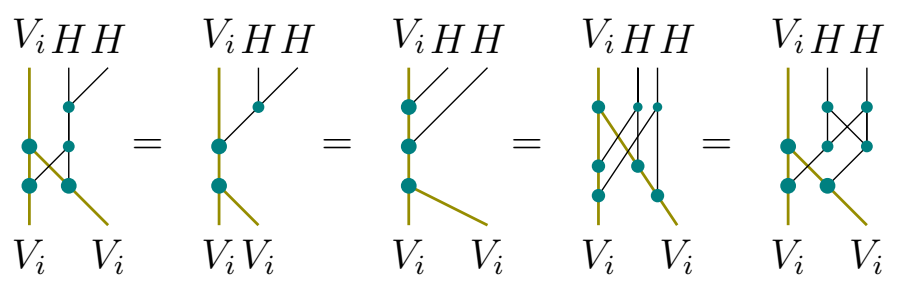




\subsection{Yetter-Drinfel'd Systems: Examples}

The examples of $H$-YD systems we give below contain usual YD modules over $H$, a priori without a UAA structure. In the following lemma we explain how to overcome this lack of structure by introducing a formal unit into a YD module:

Lemma 3.7. Let $(M, \lambda, \delta)$ be a YD module over a UAA and coUAA $H$ in a symmetric additive category $\mathcal{C}$. This YD module structure can be extended to $\widetilde{M}:=M \oplus \mathbf{I}$ as follows:

$$
\begin{array}{rlrl}
\left.\widetilde{\lambda}\right|_{H \otimes \mathbf{I}} & :=\varepsilon_{H}, & \left.\widetilde{\lambda}\right|_{H \otimes M} & :=\lambda, \\
\left.\widetilde{\delta}\right|_{\mathbf{I}} & :=\nu_{H}, & \left.\widetilde{\delta}\right|_{M}:=\delta .
\end{array}
$$

Moreover, combined with the trivial UAA structure on $\widetilde{M}$ :

$$
\left.\mu\right|_{M \otimes M}=0,\left.\quad \quad \mu\right|_{\mathbf{I} \otimes \widetilde{M}}=\left.\mu\right|_{\widetilde{M} \otimes \mathbf{I}}=\operatorname{Id}_{\widetilde{M}}, \quad \quad \nu=\operatorname{Id}_{\mathbf{I}}
$$

this extended YD module structure turns $M$ into an $H$-YD module algebra.

Proof. Direct verifications.

We need $\mathcal{C}$ to be additive in order to ensure the existence of direct sums and zero morphisms. Our favorite category Vect $_{\mathrm{k}}$ is additive.

In what follows we mostly work with YD modules admitting moreover a compatible UAA structure; the lemma shows that this assumption does not reduce the generality of our constructions.

Everything is now ready for our main example of $H$-YD systems. Recall Sweedler's notation (31) and (32).

Proposition 3.8. Let $H$ be a finite-dimensional $\mathbb{k}$-linear bialgebra, and let $M_{1}, \ldots, M_{r}$ be Yetter-Drinfel'd module algebras over $H$. Consider $H$ itself as a UAA via morphisms $\mu_{H}$ and $\nu_{H}$ and as an $H$-comodule via $\Delta_{H}$. Further, consider its dual space $H^{*}$ as a UAA via dual morphisms $\left(\Delta_{H}\right)^{*}$ and $\left(\varepsilon_{H}\right)^{*}$ and as an $H$-module via

$$
h \cdot l:=l_{(1)}(h) l_{(2)} \quad \forall h \in H, l \in H^{*}
$$

(cf. Figure 20). These structures turn the family $\left(H, M_{1}, \ldots, M_{r}, H^{*}\right)$ into an $H$-YD system.

Figure 20. The action of $H$ on $H^{*}$.

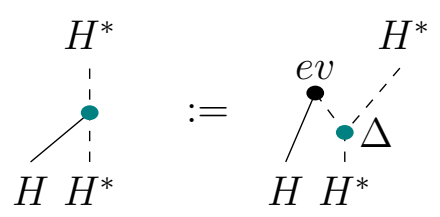

Proof. Almost all axioms from Definition 3.3 are automatic. One should check only the following points:

$\rightarrow$ the $H$-comodule structure $\Delta_{H}$ on $H$ is compatible with the UAA structure $\left(\mu_{H}, \nu_{H}\right)$;

$\rightarrow$ the map Equation (40) indeed defines an $H$-action on $H^{*}$; 
$\rightarrow$ the $H$-module structure Equation (40) on $H^{*}$ is compatible with the UAA structure $\left(\left(\Delta_{H}\right)^{*},\left(\varepsilon_{H}\right)^{*}\right)$.

These straightforward verifications use bialgebra axioms and the definition of dual morphisms. They are easily effectuated using the graphical calculus.

Applying Theorem 5 to the $H$-YD system from Proposition 3.8, and choosing braiding component $\sigma_{A s s}$ for $H^{*}$ and the $M_{i}$ 's, and its right version $\sigma_{A s s}^{r}$ for $H$ (cf. Remark 3.5), one obtains the following braided system:

Corollary 3.9. In the settings of Proposition 3.8, the system $\left(H, M_{1}, \ldots, M_{r}, H^{*}\right)$ can be endowed with the following braiding:

$$
\begin{aligned}
\sigma_{H, H} & :=\sigma_{A s s}^{r}(H)=\mu_{H} \otimes \nu_{H}: \\
\sigma_{H^{*}, H^{*}} & :=\sigma_{A s s}\left(H^{*}\right)=\left(\varepsilon_{H}\right)^{*} \otimes\left(\Delta_{H}\right)^{*}: \\
\sigma_{H, H^{*}} & :=c_{H, H^{*}}^{Y D}: \\
\sigma_{M_{i}, M_{i}} & :=\sigma_{A s s}\left(M_{i}\right)=\nu_{i} \otimes \mu_{i}: \\
\sigma_{M_{i}, M_{j}} & :=c_{M_{i}, M_{j}}^{Y D}: \\
\sigma_{H, M_{i}} & :=c_{H, M_{i}}^{Y D}: \\
\sigma_{M_{i}, H^{*}} & :=c_{M_{i}, H^{*}}^{Y D}:
\end{aligned}
$$$$
h_{1} \otimes h_{2} \mapsto h_{1} h_{2} \otimes \mathbf{1}_{H},
$$$$
l_{1} \otimes l_{2} \mapsto \varepsilon_{H} \otimes l_{1} l_{2},
$$

$$
\begin{aligned}
h \otimes l & \mapsto l_{(1)}\left(h_{(2)}\right) l_{(2)} \otimes h_{(1)}, \\
m_{1} \otimes m_{2} & \mapsto \mathbf{1}_{M_{i}} \otimes m_{1} m_{2}, \\
m \otimes n & \mapsto m_{(1)} n \otimes m_{(0)}, \\
h \otimes m & \mapsto h_{(2)} m \otimes h_{(1)}, \\
m \otimes l & \mapsto l_{(1)}\left(m_{(1)}\right) l_{(2)} \otimes m_{(0)} .
\end{aligned}
$$

Notations analogous to those from Theorem 4 are used here.

See Figures 9 and 15 for a graphical presentation of the components of the braiding from the corollary. Notation $\bar{\sigma}_{Y D A l g}$ will further be used for this braiding.

The simplest particular cases of the corollary already give interesting examples:

Example 3.10. In the extreme case $r=0$ one gets the braiding on the system $\left(H, H^{*}\right)$ studied in [8], where it was shown that:

$\rightarrow$ it encodes the bialgebra structure (e.g., the cYBE on $H \otimes H \otimes H^{*}$ or on $H \otimes H^{*} \otimes H^{*}$ is equivalent to the main bialgebra axiom (15));

$\rightarrow$ it allows to construct a fully faithful functor

$$
{ }^{*} \text { bialg }{ }^{*} \text { BrSyst }_{2}^{\bullet \bullet}
$$

where *bialg is the category of finite-dimensional bialgebras and bialgebra isomorphisms (hence notation ${ }^{*}$ ) in Vect $_{\mathbb{k}}$, and ${ }^{*}$ BrSyst $_{2}^{\bullet \bullet}$ is the category of rank 2 braided systems and their isomorphisms in $\operatorname{Vect}_{\mathbb{k}}$, endowed with some additional structure (hence notation ${ }^{\bullet \bullet}$ );

$\rightarrow$ the invertibility of the component $\sigma_{H, H^{*}}$ of this braiding is equivalent to the existence of the antipode for $H$;

$\rightarrow$ braided modules over this system (cf. [8] for a definiton) are precisely Hopf modules over $H$;

$\rightarrow$ the braided homology theory for this system (cf. Section 3.7) includes the Gerstenhaber-Schack bialgebra homology, defined in [20]. 
Example 3.11. The $r=1$ case gives a braiding on the system $\left(H, M, H^{*}\right)$ for a YD module algebra $M$ over $H$. Now observe that one can substitute the $\sigma_{M, M}=\sigma_{A s s}(M)$ component of $\bar{\sigma}_{Y D A l g}$ with the trivial one, $\sigma_{M, M}=\operatorname{Id}_{M \otimes M}$, since the instances of the cYBE involving two copies of $M$ (which are the only instances affected by the modification of $\sigma_{M, M}$ ) become automatic. Moreover, with this new $\sigma_{M, M}$ the UAA structure on $M$ is no longer necessary. One thus obtains a braiding on $\left(H, M, H^{*}\right)$ for any $H$-YD module $M$, which coincides with the one in Theorem 4 . This gives an alternative proof of that theorem.

Example 3.12. If $M$ is just a module algebra, one can extract a braided system $(H, M)$ from the construction of the previous example. Studying braided differentials for this system (cf. Section 3.7), one recovers the deformation bicomplex of module algebras, introduced by D. Yau in [21]. Note that in this example $H$ need not be finite-dimensional.

Example 3.13. The argument from example 3.11 also gives a braiding on the system $\left(H, M_{1}, \ldots, M_{r}, H^{*}\right)$ for $H$-YD modules $M_{1}, \ldots, M_{r}$, a priori without UAA structures. This braiding is obtained by replacing all the $\sigma_{M_{i}, M_{i}}$ 's from Corollary 3.9 with the trivial ones, $\sigma_{M_{i}, M_{i}}=\operatorname{Id}_{M_{i} \otimes M_{i}}$. It is denoted by $\bar{\sigma}_{Y D}$.

\subsection{Yetter-Drinfel'd Systems: Properties}

Now let us study several properties of the braiding from Corollary 3.9, namely its functoriality and the precision of encoding YD module algebra axioms.

Proposition 3.14. In the settings of Proposition 3.8, one has a faithful functor

$$
\begin{aligned}
&\left({ }_{H} \text { YDAlg }^{H}\right)^{\times r} \stackrel{i_{b r}}{\longleftrightarrow \text { BrSyst }_{r+2}} \\
& \bar{M}=\left(M_{1}, \ldots, M_{r}\right) \longmapsto\left(H, \bar{M}, H^{*} ; \bar{\sigma}_{Y D A l g}\right) \\
& \bar{f}=\left(f_{i}: M_{i} \rightarrow N_{i}\right)_{1 \leqslant i \leqslant r} \longmapsto\left(\operatorname{Id}_{H}, \bar{f}, \operatorname{Id}_{H^{*}}\right)
\end{aligned}
$$

Moreover, if a braided morphism from $i_{b r}(\bar{M})$ to $i_{b r}(\bar{N})$ has the form $\left(\operatorname{Id}_{H}, \bar{f}, \operatorname{Id}_{H^{*}}\right)$ and if the $f_{i}$ 's respect units (in the sense of Equation (7)), then all the $f_{i}: M_{i} \rightarrow N_{i}$ are morphisms in ${ }_{H} \mathbf{Y D A l g}^{H}$.

Proof. Corollary 3.9 says that the functor is well defined on objects. It remains to study the compatibility condition (29) for the collection $\left(\operatorname{Id}_{H}, \bar{f}, \operatorname{Id}_{H^{*}}\right)$ and each component of the braiding $\bar{\sigma}_{Y D A l g}$.

1. On $H \otimes H, H \otimes H^{*}$ and $H^{*} \otimes H^{*}$ condition (29) trivially holds true.

2. On $M_{i} \otimes M_{i}$, condition (29) reads

$$
\left(f_{i} \otimes f_{i}\right) \circ\left(\nu_{M_{i}} \otimes \mu_{M_{i}}\right)=\left(\nu_{N_{i}} \otimes \mu_{N_{i}}\right) \circ\left(f_{i} \otimes f_{i}\right)
$$

which, due to Equation (7), becomes

$$
\nu_{N_{i}} \otimes\left(f_{i} \circ \mu_{M_{i}}\right)=\left(\nu_{N_{i}} \otimes \mu_{N_{i}}\right) \circ\left(f_{i} \otimes f_{i}\right)
$$

But this is equivalent to $f_{i}$ respecting multiplication:

$$
f_{i} \circ \mu_{M_{i}}=\mu_{N_{i}} \circ\left(f_{i} \otimes f_{i}\right)
$$

(compose with $\mu_{N_{i}}$ on the left to get the less evident application), i.e., in the presence of Equation (7), to $f_{i}$ being a UAA morphism. 
3. On $H \otimes M_{i}$, condition (29) reads

$$
\left(f_{i} \otimes \operatorname{Id}_{H}\right) \circ c_{H, M_{i}} \circ\left(\operatorname{Id}_{H} \otimes \lambda_{M_{i}}\right) \circ\left(\Delta_{H} \otimes \operatorname{Id}_{M_{i}}\right)=c_{H, N_{i}} \circ\left(\operatorname{Id}_{H} \otimes \lambda_{N_{i}}\right) \circ\left(\Delta_{H} \otimes \operatorname{Id}_{N_{i}}\right) \circ\left(\operatorname{Id}_{H} \otimes f_{i}\right)
$$

which simplifies as

$$
\left(\operatorname{Id}_{H} \otimes\left(f_{i} \circ \lambda_{M_{i}}\right)\right) \circ\left(\Delta_{H} \otimes \operatorname{Id}_{M_{i}}\right)=\left(\operatorname{Id}_{H} \otimes \lambda_{N_{i}}\right) \circ\left(\operatorname{Id}_{H \otimes H} \otimes f_{i}\right) \circ\left(\Delta_{H} \otimes \operatorname{Id}_{M_{i}}\right)
$$

This is equivalent to $f_{i}$ respecting the $H$-module structure:

$$
\left(f_{i} \circ \lambda_{M_{i}}\right)=\lambda_{N_{i}} \circ\left(\operatorname{Id}_{H} \otimes f_{i}\right)
$$

(compose with $\varepsilon \otimes \operatorname{Id}_{N_{i}}$ on the left to get the less evident application).

4. Similarly, on $M_{i} \otimes H^{*}$ condition (29) is equivalent to $f_{i}$ respecting the $H$-comodule structure.

5. Condition (29) holds true on $M_{i} \otimes M_{j}, i<j$, if $f_{j}$ respects the $H$-module structures and $f_{i}$ respects the $H$-comodule structures.

The reader is advised to draw diagrams in order to better follow the proof.

This analysis shows that $i_{b r}$ is well defined on morphisms. Moreover, it shows that if a braided morphism from $i_{b r}(\bar{M})$ to $i_{b r}(\bar{N})$ has the form $\left(\operatorname{Id}_{H}, \bar{f}, \operatorname{Id}_{H^{*}}\right)$ and if the $f_{i}$ 's respect units, then all the $f_{i}: M_{i} \rightarrow N_{i}$ are simultaneously morphisms of UAAs (point 2 above), of $H$-modules (point 3) and of $H$-comodules (point 4), which precisely means that they are morphisms in ${ }_{H} \mathbf{Y D A l g}^{H}$.

The faithfulness of the functor is tautological.

Remark 3.15. The second statement of the proposition allows to call the functor $i_{b r}$ "essentially full". A precise description of $(\alpha, \bar{f}, \beta) \in \operatorname{Hom}_{\mathbf{B r S y s t}_{r+2}}\left(i_{b r}(\bar{M}), i_{b r}(\bar{N})\right)$ can be obtained using the results recalled in Example 3.10. Namely, if one imposes some additional restrictions ( $\alpha$ and $\beta$ should be invertible and respect the units, the $f_{i}$ 's should respect units as well), then

$\rightarrow \alpha$ is a bialgebra automorphism, and coinsides with $\left(\beta^{-1}\right)^{*}$;

$\rightarrow$ each $f_{i}$ is a UAA morphism;

$\rightarrow \alpha$ and the $f_{i}$ 's are compatible with the YD structures on the $M_{i}$ 's and $N_{i}$ 's:

$$
\begin{aligned}
f_{i} \circ \lambda_{M_{i}} & =\lambda_{N_{i}} \circ\left(\alpha \otimes f_{i}\right): H \otimes M_{i} \rightarrow N_{i} \\
\delta_{N_{i}} \circ f_{i} & =\left(f_{i} \otimes \alpha\right) \circ \delta_{M_{i}}: M_{i} \rightarrow N_{i} \otimes H
\end{aligned}
$$

Remark 3.16. Following Example 3.13, one gets a similar "essentially full" and faithful functor

$$
\begin{aligned}
&\left({ }_{H} \mathbf{Y D}^{H}\right)^{\times r} \stackrel{i_{b r}}{\longmapsto} \text { BrSyst }_{r+2} \\
& \bar{M}=\left(M_{1}, \ldots, M_{r}\right) \longmapsto\left(H, \bar{M}, H^{*} ; \bar{\sigma}_{Y D}\right) \\
& \bar{f}=\left(f_{i}: M_{i} \rightarrow N_{i}\right)_{1 \leqslant i \leqslant r} \longmapsto\left(\operatorname{Id}_{H}, \bar{f}, \operatorname{Id}_{H^{*}}\right)
\end{aligned}
$$

We next show that each instance of the cYBE for the braiding $\bar{\sigma}_{Y D A l g}$ corresponds precisely to an axiom from the Definition 3.2 of YD module algebra structure, modulo some minor normalization constraints. We thus obtain a "braided" interpretation of each of these axioms, extending the classical "braided" interpretation of the YD compatibility condition (YD) (Corollary 2.11). 
Proposition 3.17. Take a finite-dimensional $\mathbb{k}$-linear bialgebra $H$, a vector space $V$ over $\mathbb{k}$, and morphisms $\mu: V \otimes V \rightarrow V, \nu: \mathbb{k} \rightarrow V, \lambda: H \otimes V \rightarrow V, \delta: V \rightarrow V \otimes H$, a priori satisfying no compatibility relations. Consider the collection of $\sigma$ 's defined in Corollary 3.9 (for $r=1)$. One has the following equivalences:

1. $c Y B E$ for $H \otimes V \otimes H^{*} \Longleftrightarrow Y D$ compatibility condition (YD) for $(V, \lambda, \delta)$.

2. $c Y B E$ for $H \otimes H \otimes V \Longleftrightarrow \lambda$ defines an $H$-module.

Here one supposes that $\nu_{H}$ acts via $\lambda$ by identity (in the sense of Equation (13)).

3. cYBE for $V \otimes H^{*} \otimes H^{*} \Longleftrightarrow \delta$ defines an $H$-comodule.

Here one supposes that $\varepsilon_{H}$ coacts via $\delta$ by identity (in the sense dual to Equation (13)).

4. $c Y B E$ for $H \otimes V \otimes V \Longleftrightarrow \lambda$ respects the multiplication $\mu$ (in the sense of Equation (36)).

Here one supposes $\lambda$ to respect $\nu$ (in the sense of Equation (38)).

5. cYBE for $V \otimes V \otimes H^{*} \Longleftrightarrow \delta$ respects the multiplication $\mu$ (in the sense of Equation (35)).

Here one supposes $\delta$ to respect $\nu$ (in the sense of Equation (37)).

6. $c Y B E$ for $V \otimes V \otimes V \stackrel{\nu \text { is a unit for } \mu}{\Longleftrightarrow}$ associativity of $\mu$.

Proof. We prove only the first equivalence here, the other points being similar.

The cYBE for $H \otimes V \otimes H^{*}$ is graphically depicted on Figure 21(a). Using the naturality of $c$, one transforms this into the diagram on Figure 21(b). Applying $\varepsilon_{H^{*}} \otimes V \otimes \varepsilon_{H}$ to both sides and using the definition of $\Delta_{H^{*}}=\mu_{H}^{*}$ in terms of $\mu_{H}$, one gets precisely (YD).

Figure 21. cYBE for $H \otimes V \otimes H^{*} \Longleftrightarrow$ (YD).

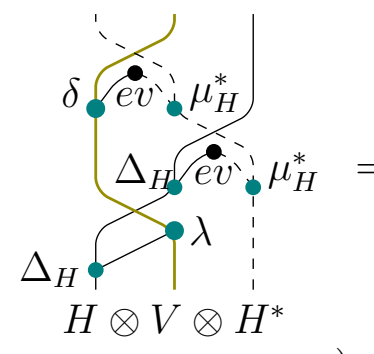

a)

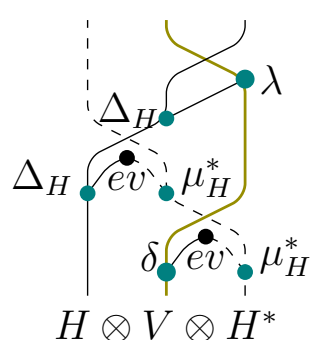

$H \otimes V \otimes H^{*}$

\subsection{Tensor Product of Yetter-Drinfel'd Modules}

The aim of this section is to explain the definition (26) and (27) of tensor product of YD modules from the braided point of view, using the braided interpretation of YD structure presented in Proposition 3.17.

Start with an easy general observation.

Lemma 3.18. Let $(\bar{V}, \bar{\sigma})$ be a rank 4 braided system in a monoidal category $\mathcal{C}$. A braiding can then be defined for the rank 3 system $\left(V_{1}, V_{2} \otimes V_{3}, V_{4}\right)$ in the following way:

$\rightarrow$ keep $\sigma_{V_{1}, V_{1}}, \sigma_{V_{1}, V_{4}}$ and $\sigma_{V_{4}, V_{4}}$ from the previous system;

$\rightarrow$ put $\sigma_{V_{2} \otimes V_{3}, V_{2} \otimes V_{3}}=\operatorname{Id}_{\left(V_{2} \otimes V_{3}\right) \otimes\left(V_{2} \otimes V_{3}\right)} ;$

$\rightarrow$ define

$$
\begin{aligned}
& \sigma_{V_{1}, V_{2} \otimes V_{3}}=\left(\operatorname{Id}_{V_{2}} \otimes \sigma_{1,3}\right) \otimes\left(\sigma_{1,2} \otimes \operatorname{Id}_{V_{3}}\right) \\
& \sigma_{V_{2} \otimes V_{3}, V_{4}}=\left(\sigma_{2,4} \otimes \operatorname{Id}_{V_{3}}\right) \otimes\left(\operatorname{Id}_{V_{2}} \otimes \sigma_{3,4}\right)
\end{aligned}
$$


Proof. The instances of the cYBE not involving $V_{2} \otimes V_{3}$ hold true since they were true in the original braided system. Those involving $V_{2} \otimes V_{3}$ at least twice are trivially true. The remaining instances (those involving $V_{2} \otimes V_{3}$ exactly once) are verified by applying the instances of the cYBE coming from the original braided system several times.

Certainly, this gluing procedure can be applied in a similar way to any two or more consecutive components in a braided system of any rank.

Applying the gluing procedure to the braided system from Example $3.13(r=2)$ and slightly rewriting the braiding obtained (using the coassociativity of $H$ and of $H^{*}$ ), one gets

Lemma 3.19. Given YD modules $M_{1}$ and $M_{2}$ over a finite-dimensional $\mathbb{k}$-linear bialgebra $H$, one has the following braiding on $\left(H, M_{1} \otimes M_{2}, H^{*}\right)$ :

$$
\begin{aligned}
& \sigma_{H, H}:=\sigma_{A s s}^{r}(H)=\mu_{H} \otimes \nu_{H} \\
& \sigma_{H^{*}, H^{*}}:=\sigma_{A s s}\left(H^{*}\right)=\left(\varepsilon_{H}\right)^{*} \otimes\left(\Delta_{H}\right)^{*} \\
& \sigma_{H, H^{*}}:=\stackrel{\circ}{c}_{H, H^{*}}^{Y D} \\
& \sigma_{M_{1} \otimes M_{2}, M_{1} \otimes M_{2}}:=\operatorname{Id}_{\left(M_{1} \otimes M_{2}\right) \otimes\left(M_{1} \otimes M_{2}\right)} \\
& \sigma_{H, M_{1} \otimes M_{2}}:=c_{H, M_{1} \otimes M_{2}} \circ\left(\operatorname{Id}_{H} \otimes{\stackrel{\lambda}{M_{1} \otimes M_{2}}}\right) \circ\left(\Delta_{H} \otimes \operatorname{Id}_{M_{1} \otimes M_{2}}\right) \\
& \sigma_{M_{1} \otimes M_{2}, H^{*}}:=c_{M_{1} \otimes M_{2}, H^{*}} \circ\left(\operatorname{Id}_{M_{1} \otimes M_{2}} \otimes \lambda_{H^{*}}\right) \circ\left({\stackrel{\circ}{M_{1} \otimes M_{2}}} \otimes \operatorname{Id}_{H^{*}}\right)
\end{aligned}
$$

where $\lambda_{H^{*}}$ denotes the action (40) of $H$ on $H^{*}$, and $\dot{\lambda}_{M_{1} \otimes M_{2}}$ and $\AA_{M_{1} \otimes M_{2}}$ are defined by Equations (26) and (27).

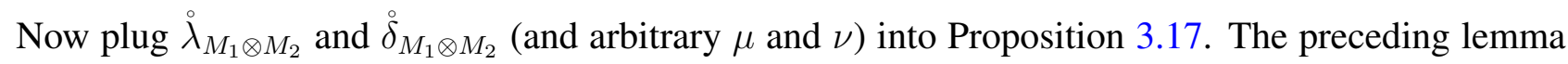
ensures the cYBE on $H \otimes\left(M_{1} \otimes M_{2}\right) \otimes H^{*}, H \otimes H \otimes\left(M_{1} \otimes M_{2}\right)$ and $\left(M_{1} \otimes M_{2}\right) \otimes H^{*} \otimes H^{*}$ (since the corresponding components of $\bar{\sigma}$ from the proposition and from the lemma coincide). The equivalences from the proposition then allow to conclude:

Corollary 3.20. Given YD modules $M_{1}$ and $M_{2}$ over a finite-dimensional $\mathbb{k}$-linear bialgebra $H$, the morphisms $\AA_{M_{1} \otimes M_{2}}$ and $\AA_{M_{1} \otimes M_{2}}$ defined by Equations (26) and (27) endow $M_{1} \otimes M_{2}$ with a YD module structure.

One thus obtains a more conceptual way of "guessing" the correct definition of tensor product for YD modules.

\subsection{Braided Homology: A Short Review}

In this section we recall the homology theory for braided systems developed in [8] (see also [13] for the rank 1, i.e., braided object, case). In the next section we apply this general theory to the braided system constructed from a YD module in Theorem 4.

We first explain what we mean by a homology theory for a braided system $(\bar{V}, \bar{\sigma})$ in an additive monoidal category $\mathcal{C}$ : 
Definition 3.21. $\rightarrow$ A degree -1 differential for a collection $\left(X_{n}\right)_{n \geqslant 0}$ of objects in $\mathcal{C}$ is a family of morphisms $\left(d_{n}: X_{n} \rightarrow X_{n-1}\right)_{n>0}$, satisfying

$$
d_{n-1} \circ d_{n}=0 \quad \forall n>1
$$

$\rightarrow$ A bidegree -1 bidifferential for a collection $\left(X_{n}\right)_{n \geqslant 0}$ of objects in $\mathcal{C}$ consists of two families of morphisms $\left(d_{n}, d_{n}^{\prime}: X_{n} \rightarrow X_{n-1}\right)_{n>0}$, satisfying

$$
d_{n-1} \circ d_{n}=d_{n-1}^{\prime} \circ d_{n}^{\prime}=d_{n-1}^{\prime} \circ d_{n}+d_{n-1} \circ d_{n}^{\prime}=0 \quad \forall n>1
$$

$\rightarrow$ An ordered tensor product for $(\bar{V}, \bar{\sigma}) \in \operatorname{BrSyst}_{r}(\mathcal{C})$ is a tensor product of the form

$$
V_{1}^{\otimes m_{1}} \otimes V_{2}^{\otimes m_{2}} \otimes \cdots \otimes V_{r}^{\otimes m_{r}}, \quad m_{i} \geqslant 0
$$

$\rightarrow$ The degree of such a tensor product is the sum $\sum_{i=1}^{r} m_{i}$.

$\rightarrow$ The direct sum of all ordered tensor products of degree $n$ is denoted by $T(\bar{V})_{n}$.

$\rightarrow$ A (bi)degree -1 (bi)differential for $(\bar{V}, \bar{\sigma})$ is a (bi)degree -1 (bi)differential for $\left(T(\bar{V})_{n}\right)_{n \geqslant 0}$.

One also needs the "braided" notion of character, restricting in particular examples to the familiar notions of character for several algebraic structures such as UAAs and Lie algebras (cf. [13]).

Definition 3.22. A braided character for $(\bar{V}, \bar{\sigma}) \in \operatorname{BrSyst}_{r}(\mathcal{C})$ is a rank $r$ braided system morphism from $(\bar{V}, \bar{\sigma})$ to $\left(\mathbf{I}, \ldots, \mathbf{I} ; \sigma_{i, j}=\operatorname{Id}_{\mathbf{I}} \forall i<j\right)$.

In other words, it is a collection of morphisms $\left(\zeta_{i}: V_{i} \rightarrow \mathbf{I}\right)_{1 \leqslant i \leqslant r}$ satisfying the compatibility condition

$$
\left(\zeta_{j} \otimes \zeta_{i}\right) \circ \sigma_{i, j}=\zeta_{i} \otimes \zeta_{j}
$$

We now exhibit a bidegree -1 bidifferential for an arbitrary braided system endowed with braided characters; see [8] for motivations, proofs, a multi-quantum shuffle interpretation and properties of this construction.

Theorem 6. Take a braided system $(\bar{V}, \bar{\sigma})$ in an additive monoidal category $\mathcal{C}$, equipped with two braided characters $\bar{\zeta}$ and $\bar{\xi}$. The families of morphisms

$$
\begin{aligned}
\left(\zeta^{\zeta} d\right)_{n} & :=\sum_{i=1}^{n}\left(\zeta_{*}\right)^{1} \circ\left(-\sigma_{*, *}\right)^{1} \circ\left(-\sigma_{*, *}\right)^{2} \circ \cdots \circ\left(-\sigma_{*, *}\right)^{i-1} \\
\left(d^{\xi}\right)_{n} & :=(-1)^{n-1} \sum_{i=1}^{n}\left(\xi_{*}\right)^{n} \circ\left(-\sigma_{*, *}\right)^{n-1} \circ \cdots \circ\left(-\sigma_{*, *}\right)^{i+1} \circ\left(-\sigma_{*, *}\right)^{i}
\end{aligned}
$$

from $T(\bar{V})_{n}$ to $T(\bar{V})_{n-1}$ define a bidegree -1 tensor bidifferential. Here the stars $*$ mean that each time one should choose the component of $\bar{\sigma}, \bar{\zeta}$ or $\bar{\xi}$ corresponding to the $V_{k}$ 's on which it acts. Further, notation (1) for superscripts is used.

Pictorially, $\left({ }^{\zeta} d\right)_{n}$ for example is a signed sum (due to the use of the negative braiding $-\bar{\sigma}$ ) of the terms presented on Figure 22. The sign can be interpreted via the intersection number of the diagram. 
Figure 22. Multi-braided left differential.

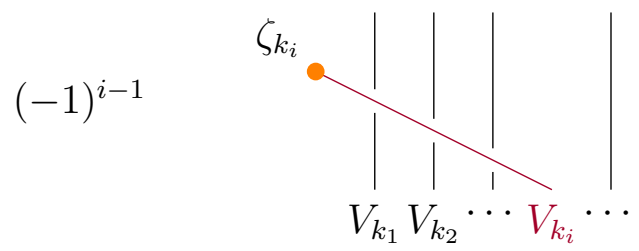

Corollary 3.23. Any $\mathbb{Z}$-linear combination of the families $\left({ }^{\zeta} d\right)_{n}$ and $\left(d^{\xi}\right)_{n}$ from the theorem is a degree -1 differential.

Definition 3.24. The (bi)differentials from the above theorem and corollary are called multi-braided.

Remark 3.25. $\rightarrow$ The constructions from the theorem are functorial.

$\rightarrow$ Applying the categorical duality to this theorem, one gets a cohomology theory for $(\bar{V}, \bar{\sigma})$.

$\rightarrow$ The braided bidifferentials can be shown to come from a structure of simplicial (or, more precisely, cubical) type.

\subsection{Braided Homology of Yetter-Drinfel'd Modules}

Let us now apply Theorem 6 to the braided system from Theorem 4. As for braided characters, we use the following ones:

Lemma 3.26. Morphisms $\left(\varepsilon_{H}: H \rightarrow \mathbb{k}, 0: M \rightarrow \mathbb{k}, 0: H^{*} \rightarrow \mathbb{k}\right)$ and $(0: H \rightarrow \mathbb{k}, 0: M \rightarrow$ $\left.\mathbb{k}, \varepsilon_{H^{*}}=\nu_{H}^{*}: H^{*} \rightarrow \mathbb{k}\right)$ are braided characters for the braided system from Theorem 4 .

These braided characters are abusively denoted by $\varepsilon_{H}$ and $\varepsilon_{H^{*}}$ in what follows.

Proof. We prove the statement for $\varepsilon_{H}$ only, the one for $\varepsilon_{H^{*}}$ being similar.

Both sides of Equation (42) are identically zero for the morphisms from the lemma, except for the case $i=j=1$, corresponding to $H \otimes H$. In this latter case, Equation (42) becomes

$$
\left(\varepsilon_{H} \otimes \varepsilon_{H}\right) \circ\left(\mu_{H} \otimes \nu_{H}\right)=\varepsilon_{H} \otimes \varepsilon_{H}
$$

which follows from the fact that $\varepsilon_{H}$ is an algebra morphism (cf. the definition of bialgebra).

In what follows, the letters $h_{i}$ always stay for elements of $H, l_{j}$ - for elements of $H^{*} ; a \in M, b \in N^{*}$; the pairing $\langle$,$\rangle is the evaluation; the multiplications \mu_{H}$ and $\Delta_{H}^{*}$ on $H$ and $H^{*}$ respectively, as well as $H$ and $H^{*}$-actions, are denoted by $\cdot$ for simplicity. We also use higher-order Sweedler's notations of type

$$
\left(\Delta_{H} \otimes \operatorname{Id}_{H}\right) \circ \Delta_{H}(h)=h_{(1)} \otimes h_{(2)} \otimes h_{(3)}, \quad \forall h \in H
$$

Further, we omit the tensor product sign when this does not lead to confusion, writing for instance $h_{1} \ldots h_{n} \in H^{\otimes n}$.

Using these notations, one can write down explicite braided differentials for a YD module: 
Proposition 3.27. Take a Yetter-Drinfel'd module $(M, \lambda, \delta)$ over a finite-dimensional $\mathbb{k}$-linear bialgebra $H$. There is a bidegree -1 bidifferential on $T(H) \otimes M \otimes T\left(H^{*}\right)$ given by

$$
\begin{aligned}
& \varepsilon_{H^{*}} d\left(h_{1} \ldots h_{n} \otimes a \otimes l_{1} \ldots l_{m}\right)= \\
& \quad(-1)^{n+1}\left\langle l_{1(1)}, a_{(1)}\right\rangle\left\langle l_{1(2)}, h_{n(2)}\right\rangle\left\langle l_{1(3)}, h_{n-1(2)}\right\rangle \ldots\left\langle l_{1(n+1)}, h_{1(2)}\right\rangle h_{1(1)} \ldots h_{n(1)} \otimes a_{(0)} \otimes l_{2} \ldots l_{m} \\
& \quad+\sum_{i=1}^{m-1}(-1)^{n+i+1} h_{1} \ldots h_{n} \otimes a \otimes l_{1} \ldots l_{i-1}\left(l_{i} \cdot l_{i+1}\right) l_{i+2} \ldots l_{m} \\
& d^{\varepsilon_{H}}\left(h_{1} \ldots h_{n} \otimes a \otimes l_{1} \ldots l_{m}\right)= \\
& \quad(-1)^{n-1}\left\langle l_{1(1)}, h_{n(m)}\right\rangle\left\langle l_{2(1)}, h_{n(m-1)}\right\rangle \ldots\left\langle l_{m(1)}, h_{n(1)}\right\rangle h_{1} \ldots h_{n-1} \otimes\left(h_{n(m+1)} \cdot a\right) \otimes l_{1(2)} \ldots l_{m(2)} \\
& \quad+\sum_{i=1}^{n-1}(-1)^{i-1} h_{1} \ldots h_{i-1}\left(h_{i} \cdot h_{i+1}\right) h_{i+2} \ldots h_{n} \otimes a \otimes l_{1} \ldots l_{m}
\end{aligned}
$$

Proof. These are just the constructions from Theorem 6 applied to the braided system from Theorem 4 and braided characters $\varepsilon_{H^{*}}$ and $\varepsilon_{H}$. Note that we restricted the differentials from $\oplus_{n=0}^{\infty} T(\bar{V})_{n}$ to $T(H) \otimes$ $M \otimes T\left(H^{*}\right)$, which is possible since both $\varepsilon_{H}$ and $\varepsilon_{H^{*}}$ are zero on $M$.

Remark 3.28. In fact the differentials ${ }^{\varepsilon_{H^{*}}} d$ and $d^{\varepsilon_{H}}$ define a double complex structure on $T(H) \otimes M \otimes$ $T\left(H^{*}\right)$, graduated by putting

$$
\overline{\operatorname{deg}}\left(H^{\otimes n} \otimes M \otimes\left(H^{*}\right)^{\otimes m}\right)=(n, m)
$$

In order to get rid of the classical contracting homotopies of type

$$
\begin{aligned}
& \bar{h} \otimes a \otimes \bar{l} \longmapsto 1_{H} \bar{h} \otimes a \otimes \bar{l} \\
& \bar{h} \otimes a \otimes \bar{l} \longmapsto \bar{h} \otimes a \otimes \bar{l} 1_{H^{*}}
\end{aligned}
$$

we now try to "cycle" this bidifferential, in the spirit of Hochschild homology for algebras or Gerstenhaber-Schack homology for bialgebras.

Concretely, take a YD module $\left(M, \lambda_{M}, \delta_{M}\right)$ and a finite-dimensional YD module $\left(N, \lambda_{N}, \delta_{N}\right)$ over $H$. Our aim is to endow the graded vector space

$$
T(H) \otimes M \otimes T\left(H^{*}\right) \otimes N^{*}
$$

with a bidifferential extending that from Proposition 3.27.

First, note that the "rainbow" duality between $H$ and $H^{*}$ (cf. (33) or Figure 14) graphically corresponds to an angle $\pi$ rotation. Since the notions of bialgebra and YD module are centrally symmetric (cf. Remark 2.10), one gets the following useful property:

Lemma 3.29. Take a finite-dimensional YD module $\left(N, \lambda_{N}, \delta_{N}\right)$ over a finite dimensional bialgebra $H$. Then $\left(N^{*}, \delta_{N}^{*}, \lambda_{N}^{*}\right)$ is a YD module over $H^{*}$. 
Further, inspired by the formulas from Proposition 3.27, consider the following morphisms from $H^{\otimes n} \otimes M \otimes\left(H^{*}\right)^{\otimes m} \otimes N^{*}$ to $H^{\otimes n-1} \otimes M \otimes\left(H^{*}\right)^{\otimes m} \otimes N^{*}$ or $H^{\otimes n} \otimes M \otimes\left(H^{*}\right)^{\otimes m-1} \otimes N^{*}$ :

$$
\begin{aligned}
& H^{*} \pi\left(h_{1} \ldots h_{n} \otimes a \otimes l_{1} \ldots l_{m} \otimes b\right)= \\
& \quad\left\langle l_{1(1)}, a_{(1)}\right\rangle\left\langle l_{1(2)}, h_{n(2)}\right\rangle\left\langle l_{1(3)}, h_{n-1(2)}\right\rangle \ldots\left\langle l_{1(n+1)}, h_{1(2)}\right\rangle h_{1(1)} \ldots h_{n(1)} \otimes a_{(0)} \otimes l_{2} \ldots l_{m} \otimes b= \\
& \quad\left\langle l_{1}, h_{1(2)} \ldots h_{n-1(2)} \cdot h_{n(2)} \cdot a_{(1)}\right\rangle h_{1(1)} \ldots h_{n(1)} \otimes a_{(0)} \otimes l_{2} \ldots l_{m} \otimes b \\
& \pi^{H^{*}}\left(h_{1} \ldots h_{n} \otimes a \otimes l_{1} \ldots l_{m} \otimes b\right)= \\
& \quad\left\langle l_{m(1)}, h_{1(1)} \cdot h_{2(1)} \ldots h_{n(1)}\right\rangle h_{1(2)} \ldots h_{n(2)} \otimes a \otimes l_{1} \ldots l_{m-1} \otimes l_{m(2)} \cdot b \\
& \pi^{H}\left(h_{1} \ldots h_{n} \otimes a \otimes l_{1} \ldots l_{m} \otimes b\right)= \\
& \quad\left\langle l_{1(1)}, h_{n(m)}\right\rangle\left\langle l_{2(1)}, h_{n(m-1)}\right\rangle \ldots\left\langle l_{m(1)}, h_{n(1)}\right\rangle h_{1} \ldots h_{n-1} \otimes\left(h_{n(m+1)} \cdot a\right) \otimes l_{1(2)} \ldots l_{m(2)} \otimes b= \\
& \quad\left\langle l_{1(1)} \cdot l_{2(1)} \ldots l_{m(1)}, h_{n(1)}\right\rangle h_{1} \ldots h_{n-1} \otimes\left(h_{n(2)} \cdot a\right) \otimes l_{1(2)} \ldots l_{m(2)} \otimes b \\
& H_{\pi}\left(h_{1} \ldots h_{n} \otimes a \otimes l_{1} \ldots l_{m} \otimes b\right)= \\
& \quad\left\langle l_{1(2)} \cdot l_{2(2)} \ldots l_{m(2)} \cdot b_{(1)}, h_{1}\right\rangle h_{2} \ldots h_{n} \otimes a \otimes l_{1(1)} \ldots l_{m(1)} \otimes b_{(0)}
\end{aligned}
$$

These applications are presented on Figure 23. We use notation

$$
\Delta^{p}:=\Delta_{H}^{p}:=\left(\Delta_{H} \otimes \operatorname{Id}_{H \otimes p-1}\right) \circ \cdots \circ\left(\Delta_{H} \otimes \operatorname{Id}_{H}\right) \circ \Delta_{H}: H \rightarrow H^{\otimes p+1} \quad \forall p \in \mathbb{N},
$$

and similarly for $\left(\mu^{*}\right)^{p}$.

Figure 23. Components of YD module homology.
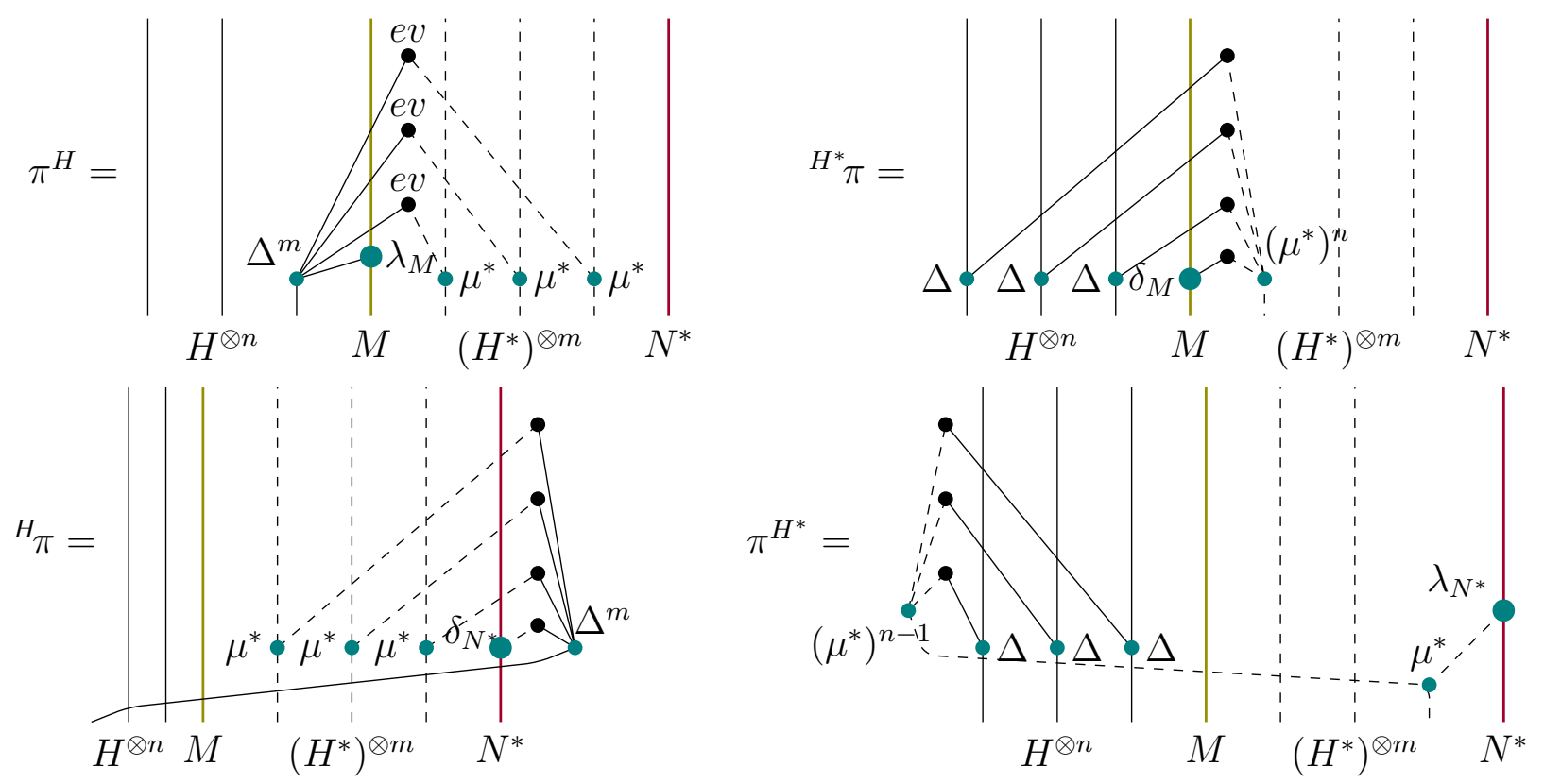

These morphisms can be interpreted in terms of "braided" adjoint actions, as it was done for the bialgebra case in [8]. 
At last, recall the classical bar and cobar differentials:

$$
\begin{aligned}
& d_{b a r}\left(h_{1} \ldots h_{n} \otimes a \otimes l_{1} \ldots l_{m} \otimes b\right)=\sum_{i=1}^{n-1}(-1)^{i} h_{1} \ldots h_{i-1}\left(h_{i} \cdot h_{i+1}\right) h_{i+2} \ldots h_{n} \otimes a \otimes l_{1} \ldots l_{m} \otimes b, \\
& d_{c o b}\left(h_{1} \ldots h_{n} \otimes a \otimes l_{1} \ldots l_{m} \otimes b\right)=\sum_{i=1}^{m-1}(-1)^{i} h_{1} \ldots h_{n} \otimes a \otimes l_{1} \ldots l_{i-1}\left(l_{i} \cdot l_{i+1}\right) l_{i+2} \ldots l_{m} \otimes b .
\end{aligned}
$$

Everything is now ready for presenting an enhanced version of Proposition 3.27:

Theorem 7. Given a YD module $M$ and a finite-dimensional YD module $N$ over a finite-dimensional bialgebra $H$ in $\mathbf{V e c t}_{\mathbb{k}}$, one has four bidegree -1 bidifferentials on $T(H) \otimes M \otimes T\left(H^{*}\right) \otimes N^{*}$, presented in the lines of Table 1.

Table 1. Bidifferential structures on $T(H) \otimes M \otimes T\left(H^{*}\right) \otimes N^{*}$.

\begin{tabular}{lcc}
\hline 1. & $d_{b a r}$ & $(-1)^{n} d_{c o b}$ \\
2. & $d_{b a r}+(-1)^{n} \pi^{H}$ & $(-1)^{n} d_{c o b}+(-1)^{n}\left(H^{*} \pi\right)$ \\
3. & $d_{b a r}+{ }^{H} \pi$ & $(-1)^{n} d_{c o b}+(-1)^{n+m} \pi^{H^{*}}$ \\
4. & $d_{b a r}+(-1)^{n} \pi^{H}+{ }^{H} \pi$ & $(-1)^{n} d_{c o b}+(-1)^{n}\left(H^{*} \pi\right)+(-1)^{n+m} \pi^{H^{*}}$ \\
\hline
\end{tabular}

The signs $(-1)^{n}$ etc. here are those one chooses on the component $H^{\otimes n} \otimes M \otimes\left(H^{*}\right)^{\otimes m} \otimes N^{*}$ of $T(H) \otimes M \otimes T\left(H^{*}\right) \otimes N^{*}$.

Substituting the graded vector space $T(H) \otimes M \otimes T\left(H^{*}\right) \otimes N^{*}$ we work in with its alternative version $\operatorname{Hom}_{\mathbb{k}}(N \otimes T(H), T(H) \otimes M)$ (as it was done for example in [22]), we obtain (the dual of a mirror version of) the deformation cohomology for YD modules, defined by F. Panaite and D. Ştefan in [14]. We have thus developed a conceptual framework for this cohomology theory, replacing case by case verifications (for instance, when proving that one has indeed a bidifferential) with a structure study, facilitated by graphical tools.

\subsection{Proof of Theorem 7}

Morphisms $d_{b a r}$ and $d_{c o b}$ are well known to be differentials; this can be easily verified by direct calculations, or using the rank 1 braided systems $\left(H, \sigma_{A s s}(H)\right)$ and $\left(H^{*}, \sigma_{A s s}\left(H^{*}\right)\right)$, cf. [13]. Further, they affect different components of $T(H) \otimes M \otimes T\left(H^{*}\right) \otimes N^{*}$ (namely, $T(H)$ and $T\left(H^{*}\right)$ ) and thus commute. The sign $(-1)^{n}$ guarantees the anti-commutation. This proves that the first line of the table contains a bidegree -1 bidifferential.

The assertion for the second line follows from Proposition 3.27, since

$$
\begin{aligned}
\varepsilon_{H^{*}} d \otimes \operatorname{Id}_{N^{*}} & =(-1)^{n+1} d_{c o b}+(-1)^{n+1}\left(H^{*} \pi\right) \\
d^{\varepsilon_{H}} \otimes \operatorname{Id}_{N^{*}} & =-d_{b a r}+(-1)^{n-1} \pi^{H}
\end{aligned}
$$

To prove the statement for the third line, note that the dual (in the sense of Lemma 3.29) version of Proposition 3.27 gives a bidifferential $\left({ }^{{ }_{H}} d, d^{\varepsilon_{H^{*}}}\right)$ on $T\left(H^{*}\right) \otimes N^{*} \otimes T(H)$, and hence (by tensoring with 
$\left.\operatorname{Id}_{M}\right)$ on $T\left(H^{*}\right) \otimes N^{*} \otimes T(H) \otimes M$. Identifying the latter space with $T(H) \otimes M \otimes T\left(H^{*}\right) \otimes N^{*}$ via the flip Equation (30) and keeping notation $\left({ }^{\varepsilon_{H}} d, d^{\varepsilon_{H^{*}}}\right)$ for the bidifferential induced on this new space, one calculates

$$
\begin{aligned}
{ }^{\varepsilon_{H}} d & =(-1)^{m+1} d_{b a r}+(-1)^{m+1}\left({ }^{H} \pi\right) \\
d^{\varepsilon_{H^{*}}} & =-d_{c o b}+(-1)^{m-1} \pi^{H^{*}}
\end{aligned}
$$

Multiplying ${ }^{\varepsilon_{H}} d$ by $(-1)^{m+1}$ and $d^{\varepsilon_{H^{*}}}$ by $(-1)^{n+1}$, and checking that this does not break the anti-commutation, one recovers the third line of the table.

We start the proof for the fourth line with a general assertion:

Lemma 3.30. Take an abelian group $(S,+, 0, x \mapsto-x)$ endowed with an operation $\cdot$ distributive with respect to + . Then, for any $a, b, c, d, e, f \in S$ such that

$$
(a+b) \cdot(d+e)=(a+c) \cdot(d+f)=a \cdot d=b \cdot f+c \cdot e=0
$$

one has

$$
(a+b+c) \cdot(d+e+f)=0
$$

Proof.

$$
(a+b+c) \cdot(d+e+f)=(a+b) \cdot(d+e)+(a+c) \cdot(d+f)-a \cdot d+(b \cdot f+c \cdot e)
$$

Now take $S=\operatorname{End}_{\mathbb{k}}\left(T(H) \otimes M \otimes T\left(H^{*}\right) \otimes N^{*}\right)$ with the usual addition and the operation composition $\varphi \circ \psi$ (for proving that the two morphisms from the fourth line of our table are differentials), or the operation $\varphi \diamond \psi:=\varphi \circ \psi+\psi \circ \varphi$ (for proving that the two morphisms anti-commute). The information from the first three lines and Lemma 3.31 allow to apply Lemma 3.30 to sextuples $(a, b, c, a, b, c)$ and $(d, e, f, d, e, f)$ (for the operation $\circ$ ), and $(a, b, c, d, e, f)$ (for the operation $\diamond)$, where

$$
\begin{array}{ll}
a:=d_{\text {bar }}, & d:=(-1)^{n} d_{c o b}, \\
b:=(-1)^{n} \pi^{H}, & e:=(-1)^{n}\left({ }^{H^{*}} \pi\right) \\
c:={ }^{H} \pi, & f:=(-1)^{n+m} \pi^{H^{*}} .
\end{array}
$$

One thus gets the fourth line of the table.

Lemma 3.31. The endomorphisms ${ }^{H^{*}} \pi, \pi^{H^{*}}, \pi^{H}$ and ${ }^{H} \pi$ of $T(H) \otimes M \otimes T\left(H^{*}\right) \otimes N^{*}$ pairwise commute.

Proof. The commutation of $\pi^{H}$ and ${ }^{H} \pi$ follows from the coassociativity of $\mu^{*}$ (this is best seen in Figure 23). The pair ${ }^{H^{*}} \pi, \pi^{H^{*}}$ is treated similarly.

The commutation of $\pi^{H}$ and ${ }^{H^{*}} \pi$ follows from their interpretation as parts of a precubical structure (cf. [13]), or by a direct computation. The pair ${ }^{H} \pi, \pi^{H^{*}}$ is treated similarly.

The case of the pair $\pi^{H}, \pi^{H^{*}}$ demands more work.

Denote by $\widehat{\pi}^{H}$ a version of $\pi^{H}$ which "forgets" the rightmost component of $H^{*}$ :

$$
\begin{aligned}
& \widehat{\pi}^{H}\left(h_{1} \ldots h_{n} \otimes a \otimes l_{1} \ldots l_{m} \otimes b\right)= \\
& \quad\left\langle l_{1(1)} \cdot l_{2(1)} \ldots l_{m-1(1)}, h_{n(1)}\right\rangle h_{1} \ldots h_{n-1} \otimes\left(h_{n(2)} \cdot a\right) \otimes l_{1(2)} \ldots l_{m-1(2)} l_{m} \otimes b
\end{aligned}
$$


Similarly, denote by $\widehat{\pi}^{H^{*}}$ a version of $\pi^{H^{*}}$ which "forgets" the rightmost component of $H$ :

$$
\begin{aligned}
& \widehat{\pi}^{H^{*}}\left(h_{1} \ldots h_{n} \otimes a \otimes l_{1} \ldots l_{m} \otimes b\right)= \\
& \quad\left\langle l_{m(1)}, h_{1(1)} \cdot h_{2(1)} \ldots . h_{n-1(1)}\right\rangle h_{1(2)} \ldots h_{n-1(2)} h_{n} \otimes a \otimes l_{1} \ldots l_{m-1} \otimes l_{m(2)} \cdot b
\end{aligned}
$$

Further, put

$$
\theta\left(h_{1} \ldots h_{n} \otimes a \otimes l_{1} \ldots l_{m} \otimes b\right)=\left\langle l_{m(1)}, h_{n(1)}\right\rangle h_{1} \ldots h_{n(2)} \otimes a \otimes l_{1} \ldots l_{m(2)} \otimes b
$$

Observe that $\theta$ is precisely the difference between the $\pi$ 's and their reduced versions $\widehat{\pi}$ :

$$
\pi^{H}=\widehat{\pi}^{H} \circ \theta, \quad \pi^{H^{*}}=\widehat{\pi}^{H^{*}} \circ \theta
$$

Moreover, one has

$$
\pi^{H} \circ \widehat{\pi}^{H^{*}}=\pi^{H^{*}} \circ \widehat{\pi}^{H}
$$

since $\pi^{H}$ and $\widehat{\pi}^{H^{*}}$ modify different components of $T(H) \otimes M \otimes T\left(H^{*}\right) \otimes N^{*}$ (the hat - (dis)appears when these morphisms switch because $\pi^{H}$ kills the rightmost copy of $H$, and $\pi^{H^{*}}$ kills the rightmost copy of $\left.H^{*}\right)$. Therefore,

$$
\pi^{H^{*}} \circ \pi^{H}=\pi^{H^{*}} \circ \widehat{\pi}^{H} \circ \theta=\pi^{H} \circ \widehat{\pi}^{H^{*}} \circ \theta=\pi^{H} \circ \pi^{H^{*}}
$$

hence the desired commutation.

The pair ${ }^{H} \pi,{ }^{H^{*}} \pi$ is treated similarly.

Remark 3.32. Lemma 3.31 actually contains more than needed for the proof of the theorem. We prefer keeping its full form and checking the commutation of all the $\left(\begin{array}{l}4 \\ 2\end{array}\right)=6$ pairs of morphisms for completeness.

\section{Acknowledgements}

The author is grateful to the Referees for the comments allowing to improve the presentation of the paper, and to Marc Rosso and Józef Przytycki for stimulating discussions.

\section{Conflicts of Interest}

The author declares no conflicts of interest.

\section{References}

1. Drinfel'd, V.G. Quantum Groups. In Proceedings of the International Congress of Mathematicians, Berkeley, CA, USA, 1986; American Mathematical Society: Providence, RI, USA, 1987; pp. 798-820.

2. Yetter, D.N. Quantum groups and representations of monoidal categories. Math. Proc. Camb. Philos. Soc. 1990, 108, 261-290. 
3. Woronowicz, S.L. Solutions of the braid equation related to a Hopf algebra. Lett. Math. Phys. 1991, 23, 143-145.

4. Montgomery, S. Hopf Algebras and Their Actions on Rings; CBMS Regional Conference Series in Mathematics; Published for the Conference Board of the Mathematical Sciences: Washington, DC, USA, 1993; Volume 82, pp. xiv+238.

5. Takeuchi, M. Survey of Braided Hopf Algebras. In New Trends in Hopf Algebra Theory (La Falda, 1999); American Mathematical Society: Providence, RI, USA, 2000; Volume 267, pp. 301-323.

6. Joyal, A.; Street, R. Braided tensor categories. Adv. Math. 1993, 102, 20-78.

7. Lebed, V. Braided Objects: Unifying Algebraic Structures and Categorifying Virtual Braids. Ph.D. Thesis, Université Paris, Paris, France, 2012.

8. Lebed, V. Braided systems, Multi-Braided Tensor Products and Bialgebra Homologies. 2013. arXiv:1305.0944. arXiv.org e-Print archive. Available online: http://arxiv.org/abs/1305.0944 (accessed on 4 September 2013).

9. Hlavatý, L.; Šnobl, L. Solution of the Yang-Baxter system for quantum doubles. Int. J. Mod. Phys. A 1999, 14, 3029-3058.

10. Nichita, F.F. New solutions for Yang-Baxter systems. Acta Univ. Apulensis Math. Inform. 2006, 11, 189-195.

11. Brzeziński, T.; Nichita, F.F. Yang-Baxter systems and entwining structures. Commun. Algebra 2005, 33, 1083-1093.

12. Lambe, L.A.; Radford, D.E. Algebraic aspects of the quantum Yang-Baxter equation. J. Algebra 1993, 154, 228-288.

13. Lebed, V. Homologies of algebraic structures via braidings and quantum shuffles. J. Algebra 2013, 391, 152-192.

14. Panaite, F.; Ştefan, D. Deformation cohomology for Yetter-Drinfel'd modules and Hopf (bi)modules. Commun. Algebra 2002, 30, 331-345.

15. MacLane, S. Categories for the Working Mathematician; Springer-Verlag: New York, NY, USA, 1971; Volume 5, pp. ix+262.

16. Kassel, C. Quantum Groups; Graduate Texts in Mathematics; Springer-Verlag: New York, NY, USA, 1995; Volume 155, pp. xii+531.

17. Faddeev, L.; Reshetikhin, N.; Takhtajan, L. Quantum Groups. In Braid Group, Knot Theory and Statistical Mechanics; World Science Publication: Teaneck, NJ, USA, 1989; Volume 9, pp. 97-110.

18. Faddeev, L.D.; Reshetikhin, N.Y.; Takhtajan, L.A. Quantization of Lie Groups and Lie algebras. In Algebraic Analysis; Academic Press: Boston, MA, USA, 1988; Volume I, pp. 129-139.

19. Radford, D.E. Solutions to the quantum Yang-Baxter equation and the Drinfel'd double. J. Algebra 1993, 161, 20-32.

20. Gerstenhaber, M.; Schack, S.D. Bialgebra cohomology, deformations, and quantum groups. Proc. Natl. Acad. Sci. USA 1990, 87, 478-481.

21. Yau, D. Deformation bicomplex of module algebras. Homol. Homotopy Appl. 2008, 10, 97-128. 
22. Mastnak, M.; Witherspoon, S. Bialgebra cohomology, pointed Hopf algebras, and deformations. J. Pure Appl. Algebra 2009, 213, 1399-1417.

(c) 2013 by the author; licensee MDPI, Basel, Switzerland. This article is an open access article distributed under the terms and conditions of the Creative Commons Attribution license (http://creativecommons.org/licenses/by/3.0/). 